GEF-TH-05-2009

\title{
The Coupling of Chern-Simons Theory to Topological Gravity
}

\author{
Camillo Imbimbd* \\ Dipartimento di Fisica, Università di Genova \\ and \\ Istituto Nazionale di Fisica Nucleare, Sezione di Genova \\ via Dodecaneso 33, I-16146, Genoa, Italy
}

\begin{abstract}
We couple Chern-Simons gauge theory to 3-dimensional topological gravity with the aim of investigating its quantum topological invariance. We derive the relevant BRST rules and Batalin-Vilkovisky action. Standard BRST transformations of the gauge field are modified by terms involving both its anti-field and the super-ghost of topological gravity. Beyond the obvious couplings to the metric and the gravitino, the BV action includes hitherto neglected couplings to the super-ghost. We use this result to determine the topological anomalies of certain higher ghost deformations of $S U(N)$ Chern-Simons theory, introduced years ago by Witten. In the context of topological strings these anomalies, which generalize the familiar framing anomaly, are expected to be cancelled by couplings of the closed string sector. We show that such couplings are obtained by dressing the closed string field with topological gravity observables.
\end{abstract}

*E-Mail: camillo.imbimbo@ge.infn.it 


\section{Introduction and Summary}

Classical Chern-Simons (CS) theory [1] on a 3-dimensional manifold $M_{3}$ is both gauge invariant and invariant under space-time diffeomorphisms, without the need to introduce a space-time metric $g_{\mu \nu}$. In the quantum theory the metric appears in the gauge-fixing term: The issue regarding topological anomalies is whether or not quantum averages depend on the chosen metric.

It was understood by Witten, in his celebrated paper [2], that quantum CS theory indeed suffers from a topological anomaly, the so-called framing anomaly. In the present work we address the question of quantum topological invariance in certain generalizations of CS gauge theory which were introduced by Witten in [4] and which are obtained by adding observables with higher ghost number to the classical CS action.

We will formulate the problem by making use of a trick which goes back to the early days of BRST renormalization methods2 and which can be explained as follows. Consider a gauge-fixed action

$$
\Gamma\left(\alpha^{i}\right)=\Gamma_{0}+S_{0} \chi\left(\alpha^{i}\right)
$$

where $\Gamma_{0}$ is the classical action, $S_{0}$ is the BRST operator, $\chi\left(\alpha^{i}\right)$ is the gaugefermion which depends on the commuting parameters $\alpha^{i}$. To simplify the notation we dropped any references to either fields or sources. The goal is understanding the (in)dependence on $\alpha^{i}$ of quantum averages, which we schematically denote as

$$
Z\left(\alpha^{i}\right)=\int \mathrm{e}^{\frac{i}{\hbar} \Gamma\left(\alpha^{i}\right)}
$$

To study this question one extends the action of $S_{0}$ to the parameters $\alpha^{i}$ by defining a new nilpotent BRST operator $\hat{S}$

$$
\hat{S} \equiv S_{0}+\beta^{i} \partial_{\alpha^{i}}
$$

where $\beta^{i}$ are anti-commuting variables. The $\hat{S}$-invariant classical action

$$
\hat{\Gamma}\left(\alpha^{i}, \beta^{i}\right) \equiv \Gamma_{0}+\hat{S} \chi\left(\alpha^{i}\right)
$$

\footnotetext{
${ }^{1}$ For a discussion which expands on the local presentation of the framing anomaly, the point of view of the present paper, see also [3].

${ }^{2}$ This idea, which appears to have been known to BRST experts for quite a long time, was rediscovered several times in different contexts. It was applied, for example, in [5] to Yang-Mills theory and in [6] to supersymmetry.
} 
defines a new "partition function" which, in general, depends on both $\alpha^{i}$ and $\beta^{i}$ :

$$
Z\left(\alpha^{i}, \beta^{i}\right)=\int \mathrm{e}^{\frac{i}{\hbar} \hat{\Gamma}\left(\alpha^{i}, \beta^{i}\right)}
$$

$Z\left(\alpha^{i}, \beta^{i}\right)$ satisfies, up to anomalies, the identity

$$
\hat{S} Z\left(\alpha^{i}, \beta^{i}\right)=0
$$

It admits an expansion in terms of the anti-commuting variables

$$
Z\left(\alpha^{i}, \beta^{i}\right)=Z^{(0)}\left(\alpha^{i}\right)+\beta^{i} Z_{i}^{(1)}\left(\alpha^{i}\right)+\beta^{i} \beta^{j} Z_{i j}^{(2)}\left(\alpha^{i}\right)+\cdots
$$

whose first term is the original quantum average one is interested in

$$
Z^{(0)}\left(\alpha^{i}\right)=Z\left(\alpha^{i}\right)
$$

The identity (1.6) translates into identities for each one of the $Z_{i_{1}, \ldots, i_{k}}^{(k)}\left(\alpha^{i}\right)$. The first one, with $k=0$, is:

$$
\beta^{i} \frac{\partial}{\partial \alpha_{i}} Z^{(0)}\left(\alpha^{i}\right)=0 \Rightarrow \frac{\partial}{\partial \alpha_{i}} Z\left(\alpha^{i}\right)=0
$$

It just states the independence of the original $Z\left(\alpha^{i}\right)$ from the gauge parameters $\alpha^{i}$. The advantage of reformulating the (classical) gauge independence of $Z\left(\alpha^{i}\right)$ as the cocyle condition (1.6) for its extension $Z\left(\alpha^{i}, \beta^{i}\right)$ is that this permits the use of powerful cohomological methods to investigate the corresponding quantum anomalies.

In traditional quantum field theory the introduction of $Z\left(\alpha^{i}, \beta^{i}\right)$ is just a technical trick: The components $Z_{i_{1}, \ldots i_{k}}^{(k)}\left(\alpha^{i}\right)$ with $k>0$ have usually no physical interest. As a matter of fact, they typically vanish, due to ghost number conservation, since the $\beta^{i}$ s carry ghost number +1 . Both in string theory and in topological field theories the situation is different since observables have generally non-vanishing positive ghost number. The classical identity (1.6) says that the components of fixed $\beta$-degree of the generalized partition function

$$
Z^{(k)}\left(\alpha^{i}, \beta^{i}\right) \equiv \beta^{i_{1}} \cdots \beta^{i_{k}} Z_{i_{1} \cdots i_{k}}^{(k)}\left(\alpha^{i}\right)
$$

should be thought of as closed $k$-forms on the manifold parameterized by the $\alpha^{i}$ :

$$
\beta^{i_{1}} \beta^{i_{2}} \cdots \beta^{i_{k}} \beta^{i_{k+1}} \partial_{i_{1}} Z_{i_{2} \cdots i_{k+1}}^{(k)}\left(\alpha^{i}\right)=0
$$


In string theory, for example, the parameters $\alpha^{i}$ are coordinates on the moduli space of Riemann surfaces and the total ghost number of the observables is such that the non-vanishing component of $Z(\alpha, \beta)$ is a top-form on the relevant moduli space [7].

In the context of CS theory, the role of the parameters $\alpha^{i}$ is played by the background metric $g_{\mu \nu}(x)$. The BRST operator $S_{0}$ is the one encoding gauge invariance of the classical action. It was therefore suggested by Witten in [4] to introduce a fermionic gravitino background $\psi_{\mu \nu}(x)$ and, in analogy with (1.3), to extend the action of the BRST operator to the backgrounds

$$
\hat{S} g_{\mu \nu}=\psi_{\mu \nu} \quad \hat{S} \psi_{\mu \nu}=0
$$

In the same paper Witten proposed also to add to the CS classical action observables with positive ghost number. In this way one would obtain nonvanishing components $Z^{(k)}$ with $k>0$ which would naturally define cohomology classes on the space of 3-dimensional metrics on the manifold $M_{3}$.

In this paper we reconsider and refine this proposal by starting from the following observation. The BRST rules (1.12) are those of topological gravity [8]. Or, more precisely, they are the "naive" BRST transformations of topological gravity. The correct ones are the equivariant ones [9, 10, 11, 12 ]

$$
\begin{aligned}
& s g_{\mu \nu}=\psi_{\mu \nu}-\mathcal{L}_{\xi} g_{\mu \nu} \quad s \psi_{\mu \nu}=\mathcal{L}_{\gamma} g_{\mu \nu}-\mathcal{L}_{\xi} \psi_{\mu \nu} \\
& s \xi^{\mu}=\gamma^{\mu}-\frac{1}{2} \mathcal{L}_{\xi} \xi^{\mu} \quad s \gamma^{\mu}=-\mathcal{L}_{\xi} \gamma^{\mu}
\end{aligned}
$$

which involve, beyond the metric and the gravitino, the reparametrization ghost $\xi^{\mu}$, the corresponding super-ghost ${ }^{3} \gamma^{\mu}$ (of ghost number +2 ) and infinitesimal diffeomorphisms $\mathcal{L}_{\xi}\left(\mathcal{L}_{\gamma}\right)$ with parameters $\xi^{\mu}\left(\gamma^{\mu}\right)$. The reason to go from (1.12) to (1.13) is to ensure that the closed forms $Z^{(k)}$ descend to globally defined forms on the quotient of the space of 3-dimensional metrics under the action of diffeomorphisms[10, 7]. This will be reviewed in Section 5 .

We find that once we extend the action of the BRST operator to the gravitational backgrounds in the equivariant way, we must - to preserve nilpotency — modify the BRST transformations in the gauge sector. We will work this out in Section 2, It turns out that the new BRST operator can be expressed in terms of an operator $S$ which writes as follows

$$
S \equiv \hat{S}+G_{\gamma}
$$

\footnotetext{
${ }^{3} \gamma^{\mu}$ can also be thought of as a "ghost-for-ghost".
} 
Here $\hat{S}$ is the "naive" extension (1.12) of the standard BRST operator of gauge theories to the gravitational backgrounds. $G_{\gamma}$ is the novel term dictated by equivariance which is at the center of our analysis. It has degree 1 in the super-ghost field $\gamma^{\mu}$ and it deforms in a non-trivial way the BRST rules of the gauge sector. $\hat{S}$ and $G_{\gamma}$ satisfy an $N=2$ supersymmetry algebra

$$
\hat{S}^{2}=G_{\gamma}^{2}=0 \quad\left\{\hat{S}, G_{\gamma}\right\}=\mathcal{L}_{\gamma}
$$

and the complete nilpotent equivariant BRST operator is simply $s=S-\mathcal{L}_{\xi}$.

An important point is that the $G_{\gamma}$-transformation of the gauge fields depends on their anti-fields. In other words, a proper off-shell formulation of $G_{\gamma}$ requires adopting the Batalin-Vilkovisky formalism.

Having deformed the BRST operator in a non-trivial way, one needs to reconsider the classical action. This is not simply given by the obvious analog of (1.4), precisely because the BRST variation of the gauge field contains its anti-field. We present the complete Batalin-Vilkovisky classical action in Section 3. The peculiar feature of this action is a term which is quadratic in the anti-fields and linear in the super-ghost field $\gamma^{\mu}$. We believe this term is new and has not been worked out before.

The somewhat surprising outcome of this analysis is that the consistent formulation of CS theory on a curved manifold entails coupling it not only to the metric and the gravitino backgrounds, but also to the super-ghost field 4 . We make a digression in Section 3.2 to understand this from the point of view of the theory in flat space. In essence, the story goes as follows. The theory in flat space has a stress-energy tensor $T_{\mu \nu}$ which is BRST-exact

$$
T_{\mu \nu}=S_{0} G_{\mu \nu}
$$

$G_{\mu \nu}$ is defined by this relation only up to $S_{0}$-exact terms. It turns out that if one takes it to be symmetric with respect to the exchange of $\mu$ and $\nu$, as appropriate for the current coupled to the gravitino, $G_{\mu \nu}$ is conserved only up to $S_{0}$-trivial terms. One can define a truly conserved super-current $\tilde{G}_{\mu \nu}$, but this necessarily has an anti-symmetric piece: While the symmetric part of $\tilde{G}_{\mu \nu}$ couples to the gravitino, the anti-symmetric part needs a vector field to couple to. This explains the necessity of the source $\gamma^{\mu}$ from the point of view of currents in flat space. Incidentally, the conserved $\tilde{G}_{\mu \nu}$, which is the

\footnotetext{
${ }^{4}$ The reparametrization ghost $\xi^{\mu}$ drops out of the gauge-fixed action, as dictated by equivariance.
} 
remnant in flat space of the BRST charge $G_{\gamma}$, is the super-current associated to the vector super-symmetry of gauge-fixed CS in flat space which was discovered years ago [15]. The coupling to topological gravity provides, in a sense, a more conceptual explanation of this flat space global symmetry.

In Section 4 we reconsider, in the equivariant context, the observables of positive ghost number put forward by Witten. We find that one can build equivariant gauge observables if one includes the anti-fields of the gauge sector. Since the observables involve anti-fields, adding them to the theory will, in general, not only modify the action but also deform the BRST transformations. We work out in some detail the case of gauge group $G=S U(N)$. For this theory we find $N-1$ "primitive" single trace observables of positive ghost number, all other observables being multi-traces of the primitive ones. The single-trace higher-ghost deformation of $S U(N)$ CS theory depends therefore on $N-1$ parameters $t_{i}$, with $i=1, \ldots N-1$, in addition to the gauge coupling constant. The corresponding $Z^{(2 p)}$ are $2 p$-forms on the moduli space of 3-dimensional metrics, which are homogeneous polynomials of degree $p$ in the $t_{i}$ 's, with $t_{i}$ of weight $i$.

In Section 6 we discuss at last the topological anomalies of the deformed CS theory, that is the failure of the forms $Z^{(2 p)}$ to be closed. Here the trick (1.3) pays off, since the problem is reduced to determining local observables of 3-dimensional topological gravity. Using methods [23, 24] which have been applied in the literature mostly in dimension 2 and 4, we find that topological gravity observables in $3 \mathrm{~d}$ - and thus CS topological anomalies - are powers of a single basic observable

$$
\mathcal{A}_{4 p}=\left(\operatorname{tr} \mathcal{R}^{2}\right)^{p} \quad p=1,2, \ldots
$$

$\mathcal{R}$ is a generalized curvature form of total (form+ghost number) fermionic degree 2, whose 2-form component is the curvature of the background metric $g_{\mu \nu}$. The $p=1$ observable is Witten's framing anomaly, the $p>1$ observables are higher-ghost generalizations of it.

The cohomological analysis does not yield the "coefficients" of the anomalies. These "coefficients" are in fact polynomials $c_{2(p-1)}\left(t_{i}\right)$ in $t_{i}$, homogeneous of degree $2(p-1)$, with $t_{i}$ of weight $i$. It is possible that the numerical coefficients of such polynomials have some interesting topological interpretation. We postpone to the future attempts to compute them explicitly.

In the last Section 7 we try to integrate our analysis with the insight of Witten [4] that $S U(N)$ CS theory on $M_{3}$ is the target space field theory of 
topological open strings propagating on the non-compact Calabi-Yau (complex) 3-manifold $X_{6}=T^{*} M_{3}$. One expects that the complete closed and open topological string theory be anomaly free. We propose a simple and natural way to introduce couplings in the closed string field theory which cancel the higher-ghost anomalies of the deformed $S U(N)$ CS theory. Following [25] we assume that the closed string field $\mathcal{K}$ be a generalized form of total (form + ghost number) degree equal to 2 . Then the coupling of the closed string field to the observables of topological gravity

$$
\Gamma_{\text {anomaly }}=\sum_{p} c_{2(p-1)}\left(t_{i}\right) \int_{X_{6}} \mathcal{K}\left(\operatorname{tr} \mathcal{R}^{2}\right)^{p}
$$

cancel the topological anomalies of the open string sector. In 26] it was checked essentially that the $p=1$ term of this sum is indeed consistent with what is known about the partition function of the topological closed string model.

Beyond the explicit computation of the anomaly polynomials $c_{2(p-1)}\left(t_{i}\right)$ there are several other problems that are left open by this work. The relation between the anomalies of $S U(N)$ deformed CS and 6-dimensional topological gravity is somewhat reminiscent of the Kontsevitch relation between large $N$ matrix theory and 2-dimensional topological gravity [27],28]. One might speculate if this analogy could lead to a combinatorial interpretation of the anomaly polynomials $c_{2(p-1)}\left(t_{i}\right)$ and to an efficient way to compute them.

It would be also important to understand how one computes, on the topological string side, amplitudes involving CS couplings with higher ghost number. We believe that playing the trick (1.3) at the level of the topological sigma model maybe useful in this regard. But the details have not been worked out. Understanding this issue might open the way to significant and possibly fruitful generalizations of the open-closed topological string duality of $[26]$.

\section{Chern-Simons Topological Structure}

Let

$$
A=A_{\mu}^{a} T^{a} d x^{\mu}
$$

be a 1 -form gauge field on a closed 3 -manifold $M_{3} . T^{a}$, with $a=1, \ldots, \operatorname{dim} G$, are generators of the Lie algebra of the gauge group $G$ which will be taken 
to be simple, connected and simply connected. The classical CS action [1] writes as follows

$$
\Gamma_{C S}=\int_{M_{3}} \operatorname{Tr}\left[\frac{1}{2} A d A+\frac{1}{3} A^{3}\right]
$$

Gauge invariance leads to the nilpotent BRST transformation rules 5

$$
S_{0} A=D c \quad S_{0} c=c^{2}
$$

where $c=c^{a} T^{a}$ is the ghost field carrying ghost number +1 and $D c \equiv$ $d c+[A, c]$ is the covariant differential.

It is useful to associate to $A$ and $c$, respectively, the anti-fields $A^{*}$ and $c^{*}$, of ghost number -1 and $-2 . A^{*}$ and $c^{*}$ are Lie algebra-valued 2 and 3 -forms:

$$
A^{*}=\left(A^{*}\right)_{\mu \nu}^{a} T^{a} d x^{\mu} d x^{\nu} \quad c^{*}=\left(c^{*}\right)_{\mu \nu \rho}^{a} T^{a} d x^{\mu} d x^{\nu} d x^{\rho}
$$

The corresponding Batalin-Vilkovisky action

$$
\Gamma_{B V}=\Gamma_{C S}[A]+\int_{M_{3}} \operatorname{Tr}\left[\left(S_{0} A\right) A^{*}+\left(S_{0} c\right) c^{*}\right]
$$

is $S_{0}$ invariant if one extends the action of $S_{0}$ to the anti-fields in the following way

$$
S_{0} A^{*}=F+\left[A^{*}, c\right] \quad S_{0} c^{*}=D A^{*}+\left[c^{*}, c\right]
$$

where the square brackets denote either commutator or anti-commutator according to the ghost numbers of the fields involved. The BRST operator $S_{0}$ defined by (2.3) and (2.6) is nilpotent on both the fields and the anti-fields.

The classical action (2.2) is invariant not just under diffeomorphisms of $M_{3}$ : it is also topological, i.e. it is independent of the 3-dimensional background metric $g_{\mu \nu}$. The gauge-fixed action necessarily depends on $g_{\mu \nu}$. The issue of topological anomalies is if quantum averages do depend on $g_{\mu \nu}$ or not.

To discuss this issue one extends the action of $S_{0}$ to the background metric6

$$
S_{0} g_{\mu \nu}=\psi_{\mu \nu} \quad S_{0} \psi_{\mu \nu}=0
$$

\footnotetext{
${ }^{5}$ We will adopt the convention that BRST operator $S_{0}$ anti-commutes with the exterior differential $d$.

${ }^{6}$ To reduce symbols proliferation, we denote the extended BRST operator with the same symbol $S_{0}$ as the original one. In the Introduction we used the symbol $\hat{S}$ for the same object.
} 
where $\psi_{\mu \nu}$ is the topological fermionic gravitino field with ghost number +1 . Such an extension of the BRST transformations for CS theory was first considered in [4].

The BRST transformation rules (2.7) are essentially those of topological gravity as it was originally defined in [8]. In this sense, to discuss topological invariance of CS gauge theory one needs to couple it to topological gravity.

Since the original paper [8], however, it has been understood [9, 10, 11, 12] that the "naive" BRST rules (2.7) must be slightly modified7. The correct BRST operator of topological gravity is the equivariant one, which, in some sense, factors out diffeomorphisms. This necessitates introducing both the anti-commuting ghost fields $\xi^{\mu}$ of ghost number +1 , associated to 3-dimensional diffeomorphisms, and its super-partner, the commuting superghost field $\gamma^{\mu}$ of ghost number +2 . Eqs. (2.7) are to be replaced by the equivariant ones

$$
\begin{aligned}
& s g_{\mu \nu}=\psi_{\mu \nu}-\mathcal{L}_{\xi} g_{\mu \nu} \quad s \psi_{\mu \nu}=\mathcal{L}_{\gamma} g_{\mu \nu}-\mathcal{L}_{\xi} \psi_{\mu \nu} \\
& s \xi^{\mu}=\gamma^{\mu}-\frac{1}{2} \mathcal{L}_{\xi} \xi^{\mu} \quad s \gamma^{\mu}=-\mathcal{L}_{\xi} \gamma^{\mu}
\end{aligned}
$$

where $\mathcal{L}_{\xi}$ denotes the Lie derivative along the vector field $\xi^{\mu}$.

Our starting and basic observation is the following. Once we switch from the "naive" $S_{0}$ to the equivariant $s$ in the gravitational background sector we must do the same in the "matter" gauge sector, i.e. in the sector generated by the quantum fields $c, A$ and by their anti-fields $c^{*}, A^{*}$. Starting from the ansatz

$$
s=S_{0}-\mathcal{L}_{\xi}+\cdots \quad \text { on the gauge sector }
$$

we determine the dots by requiring nilpotency

$$
s^{2}=0 \quad \text { on all fields }
$$

We obtain in this way to the following BRST action on the gauge sector 8

$$
s c=c^{2}-\mathcal{L}_{\xi} c-i_{\gamma}(A)
$$

\footnotetext{
${ }^{7}$ We review the reason for this in Section 5 ,

${ }^{8}$ BRST rules which combine gauge symmetry and topological gravity transformations had been derived, with different methods and motivations, in [13. The BRST rules of [13 are quite similar, although not identical, to the ones we present here. In particular the $i_{\gamma}(A)$ term in the BRST variation of the $c$ ghost already makes its appearance in [13. However, since the focus of that work is on higher-dimensional theories, the details of the BRST action on gauge fields seem different than ours. More importantly, anti-fields are not considered in 13. The structure of the anti-field sector specific to 3-dimensions is essential for the off-shell nilpotency of the transformations (2.11).
} 


$$
\begin{aligned}
& s A=D c-\mathcal{L}_{\xi} A-i_{\gamma}\left(A^{*}\right) \\
& s A^{*}=F-\mathcal{L}_{\xi} A^{*}-\left[A^{*}, c\right]-i_{\gamma}\left(c^{*}\right) \\
& s c^{*}=D A^{*}-\mathcal{L}_{\xi} c^{*}+\left[c^{*}, c\right]
\end{aligned}
$$

where $i_{\gamma}$ acts on forms and denotes the contraction with the vector field $\gamma^{\mu}$ :

$$
i_{\gamma}(A) \equiv \gamma^{\mu} A_{\mu} \quad \text { etc. }
$$

The relevant notion of BRST cohomology in topological gravity is the equivariant one9. This means that physical observables are associated to the cohomology of $s$ on the algebra generated by all fields with the exclusion of the reparametrization ghosts $\xi^{\mu}$. When restricting oneself to such a space it is useful to introduce the operator

$$
S \equiv s+\mathcal{L}_{\xi}
$$

Nilpotency of $s$ is equivalent to the following property of $S$

$$
S^{2}=\mathcal{L}_{\gamma} \quad \text { on all fields but } \xi^{\mu}
$$

Hence, $S$ is nilpotent when acting on reparametrization invariants functionals which are also independent of $\xi^{\mu}$. The action of $S$, both on quantum fields and classical backgrounds, is

$$
\begin{array}{cl}
S c=c^{2}-i_{\gamma}(A) & S A=D c-i_{\gamma}\left(A^{*}\right) \\
S A^{*}=F+\left[A^{*}, c\right]-i_{\gamma}\left(c^{*}\right) & S c^{*}=D A^{*}+\left[c^{*}, c\right] \\
S g_{\mu \nu}=\psi_{\mu \nu} & S \psi_{\mu \nu}=\mathcal{L}_{\gamma} g_{\mu \nu} \\
S \gamma^{\mu}=0 &
\end{array}
$$

$S$ can be decomposed as

$$
S=S_{0}+G_{\gamma}
$$

where $S_{0}$ is the "naive" nilpotent BRST operator defined in (2.3), (2.6), (2.7) while $G_{\gamma}$ is the nilpotent operator, linear in the field $\gamma^{\mu}$, defined by

$$
\begin{array}{llrl}
G_{\gamma} c=-i_{\gamma}(A) & G_{\gamma} A=-i_{\gamma}\left(A^{*}\right) \quad G_{\gamma} A^{*}=-i_{\gamma}\left(c^{*}\right) & G_{\gamma} c^{*}=0 \\
G_{\gamma} g_{\mu \nu}=0 & G_{\gamma} \psi_{\mu \nu}=\mathcal{L}_{\gamma} g_{\mu \nu} & G_{\gamma} \gamma^{\mu}=0 &
\end{array}
$$

\footnotetext{
${ }^{9}$ See the Appendix $\mathrm{A}$ for a review of the relevant material.
} 
$S_{0}$ and $G_{\gamma}$ generate a supersymmetric algebra

$$
S_{0}^{2}=0 \quad G_{\gamma}^{2}=0 \quad\left\{S_{0}, G_{\gamma}\right\}=\mathcal{L}_{\gamma}
$$

This $N=2$ BRST algebra exposes the topological nature of CS theory: Infinitesimal diffeomorphisms are expressed as commutator of $S_{0}$ a second BRST symmetry charge, $G_{\gamma}$.

\section{The BV Action}

In this section we construct the Batalin-Vilkovisky action 10 associated to the equivariant $s$. The BV action relative to the "naive" BRST operator $S_{0}$ is linear in the anti-fields:

$$
\Gamma_{0}=\Gamma_{C S}[A]+\int_{M_{3}} \operatorname{Tr}\left[\left(S_{0} A\right) A^{*}+\left(S_{0} c\right) c^{*}+\left(S_{0} g_{\mu \nu}\right)\left(g^{*}\right)^{\mu \nu}\right]
$$

where $\left(g^{*}\right)^{\mu \nu}$ is the anti-field associated to $g_{\mu \nu}$ transforming as a tensorial density under diffeomorphisms. This "flat space" BV action is equivalent to the one first presented in [21] and also discussed in [22].

To construct the BV action associated to the equivariant $s$ we start therefore from the analogous expression

$$
\Gamma_{B V}=\Gamma_{C S}+\sum_{\Phi} \int_{M_{3}}(s \Phi) \Phi^{*}+\cdots
$$

where we denote by $\Phi$ the collection of all fields and by $\Phi^{*}$ their anti-fields, both those of the gauge sector (i.e. $A, A^{*}, c, c^{*}$ ) and those of the gravitational sector (i.e. $\left.g_{\mu \nu}, \psi_{\mu \nu}, \xi^{\mu}, \gamma^{\mu}\right)$. Explicitly,

$$
\begin{aligned}
\Gamma_{B V} & =\Gamma_{0}-\int_{M_{3}} \operatorname{Tr}\left[\mathcal{L}_{\xi} A A^{*}+\mathcal{L}_{\xi} c c^{*}+\right. \\
& +\left(\mathcal{L}_{\xi} g_{\mu \nu}\right)\left(g^{*}\right)^{\mu \nu}+\left(\mathcal{L}_{\xi} \psi_{\mu \nu}\right)\left(\psi^{*}\right)^{\mu \nu}+\frac{1}{2}\left(\mathcal{L}_{\xi} \xi^{\mu}\right) \xi_{\mu}^{*}+\left(\mathcal{L}_{\xi} \gamma^{\mu}\right) \gamma_{\mu}^{*}+ \\
& \left.+i_{\gamma}\left(A^{*}\right) A^{*}+i_{\gamma}(A) c^{*}-\left(\mathcal{L}_{\gamma} g_{\mu \nu}\right)\left(\psi^{*}\right)^{\mu \nu}-\gamma^{\mu} \xi_{\mu}^{*}\right]+\cdots
\end{aligned}
$$

where we introduced the anti-fields relative to both $\xi^{\mu}$ and $\gamma^{\mu}$.

\footnotetext{
${ }^{10}$ For a short review of the BV formalism, see for example [14, of which we adopt the notation.
} 
However, as the dots in the r.h.s. of (3.2) indicate, this action is not invariant under $s$. This can be traced back to the term proportional to the anti-field $A^{*}$ in the BRST variation of $A$, giving rise to the term $-\operatorname{Tr} i_{\gamma}\left(A^{*}\right) A^{*}$ in the action, which is quadratic in the anti-fields. To achieve invariance we need to half the coefficient of such a term; we do so by adding to the action an identical term with coefficient $1 / 2$

$$
\begin{aligned}
\Gamma_{B V} & =\Gamma_{C S}+\int_{M_{3}} \operatorname{Tr}\left[\sum_{\Phi}(s \Phi) \Phi^{*}+\frac{1}{2} i_{\gamma}\left(A^{*}\right) A^{*}\right]= \\
& =\Gamma_{0}-\int_{M_{3}} \operatorname{Tr}\left[\left(\mathcal{L}_{\xi} A\right) A^{*}+\left(\mathcal{L}_{\xi} c\right) c^{*}+\right. \\
& +\left(\mathcal{L}_{\xi} g_{\mu \nu}\right)\left(g^{*}\right)^{\mu \nu}+\left(\mathcal{L}_{\xi} \psi_{\mu \nu}\right)\left(\psi^{*}\right)^{\mu \nu}+\frac{1}{2}\left(\mathcal{L}_{\xi} \xi^{\mu}\right) \xi_{\mu}^{*}+\left(\mathcal{L}_{\xi} \gamma^{\mu}\right) \gamma_{\mu}^{*}+ \\
& \left.+\frac{1}{2} i_{\gamma}\left(A^{*}\right) A^{*}+i_{\gamma}(A) c^{*}-\left(\mathcal{L}_{\gamma} g_{\mu \nu}\right)\left(\psi^{*}\right)^{\mu \nu}-\gamma^{\mu} \xi_{\mu}^{*}\right]
\end{aligned}
$$

This is the BV action invariant under the equivariant $s . \Gamma_{0}$ is the BV CS gauge action associated to the "naive" BRST operator $S_{0}$. The other terms in $\Gamma$ describe the couplings of CS theory to both the ghost and the superghost of topological gravity. The latter are required for the equivariance of the theory. As far as we know, the couplings to the super-ghost $\gamma^{\mu}$ are new and had not been considered before.

On can check directly that the BV action (3.3) reproduces the equivariant BRST transformations (2.8) and (2.11) via the known formula:11

$$
s \Phi=\left(\Gamma_{B V}, \Phi\right)=\frac{\delta^{R} \Gamma_{B V}}{\delta \Phi^{*}} \quad s \Phi^{*}=\left(\Gamma_{B V}, \Phi^{*}\right)=\frac{\delta^{R} \Gamma_{B V}}{\delta \Phi}
$$

where we introduced the BV bracket

$$
(F, G) \equiv \sum_{\Phi} \frac{\delta^{R} F}{\delta \Phi} \frac{\delta^{L} G}{\delta \Phi^{*}}+\frac{\delta^{R} F}{\delta \Phi^{*}} \frac{\delta^{L} G}{\delta \Phi}
$$

For example, the factor $1 / 2$ in front of the term in the action quadratic in the anti-field $A^{*}$ ensures that the derivative of the action with respect to $A^{*}$

\footnotetext{
${ }^{11}$ Some signs in formulas (3.4) and (3.5) are different than how they are usually written. This is so since in our convention the BRST operator anti-commutes with the exterior differential. With this choice, $A, c$ have both odd total fermion number, as well as their anti-fields and $\Gamma_{B V}$. This convention will make formulas in Section 4 look nicer.
} 
coincides with the BRST transformation rule for $A$

$$
s A=\frac{\delta^{R} \Gamma_{B V}}{\delta A^{*}}=-i_{\gamma}\left(A^{*}\right)+\cdots
$$

As dictated by the BV formalism, both the nilpotence of $s$ and the BRST invariance of $\Gamma$ are captured by the single equation

$$
\left(\Gamma_{B V}, \Gamma_{B V}\right)=0
$$

\subsection{The gauge-fixed action}

Gauge-fixing is achieved by introducing a suitable functional $\chi[\Phi]$ of the fields. The gauge-fixed action $\Gamma_{\chi}$ is then given by the formula

$$
\Gamma_{\chi}[\Phi]=\Gamma_{B V}\left[\Phi, \Phi^{*}=\frac{\delta \chi}{\delta \Phi}\right]
$$

and the gauge-fixed BRST operator is

$$
s_{\chi} \Phi=\left.(s \Phi)\right|_{\Phi^{*}=\frac{\delta \chi}{\delta \Phi}}
$$

The square of $s_{\chi}$ is

$$
s_{\chi}^{2} \Phi=-\left.\sum_{\Phi^{\prime}} \frac{\delta^{R} \Gamma_{\chi}}{\delta \Phi^{\prime}} \frac{\delta^{L}(s \Phi)}{\delta\left(\Phi^{\prime}\right)^{*}}\right|_{\Phi^{*}=\frac{\delta \chi}{\delta \Phi}}
$$

Therefore, when the BV action $\Gamma_{B V}\left[\Phi, \Phi^{*}\right]$ is not linear in the anti-fields, $s_{\chi}$ is nilpotent only on the (gauge-fixed) shell.

The gauge-fixed action is $s_{\chi}$-invariant (off-shell, of course):

$$
s_{\chi} \Gamma_{\chi}=0
$$

The gauge-fermion $\chi[\Phi]$ must be chosen in such a way that all fields have invertible kinetic terms. To achieve this, it is typically necessary to introduce more fields, beyond gauge and ghost (anti)fields. For CS theory we must add both the anti-ghost $b$ and the lagrangian multiplier $\Lambda$

$$
b=T^{a} b_{\mu \nu \rho}^{a} d x^{\mu} d x^{\nu} d x^{\rho} \quad \Lambda=T^{a} \Lambda_{\mu \nu \rho}^{a} d x^{\mu} d x^{\nu} d x^{\rho}
$$


which are 3 -forms with values in the Lie algebra of the gauge group. Their BRST transformation laws are

$$
s b=\Lambda-\mathcal{L}_{\xi} b \quad s \Lambda=\mathcal{L}_{\gamma} b-\mathcal{L}_{\xi} \Lambda
$$

From now on, we will denote by $\Phi$ the collection of all fields, coming from the gauge, the gravitational and anti-ghost sectors.

From (3.3) we obtain the CS gauge-fixed action

$$
\Gamma_{\chi}=\Gamma_{C S}[A]+s_{\chi}(\chi[\Phi])-\frac{1}{2} \int_{M_{3}} \operatorname{Tr}\left(i_{\gamma}\left(\frac{\delta \chi}{\delta A}\right) \frac{\delta \chi}{\delta A}\right)
$$

and the gauge-fixed BRST transformations

$$
\begin{aligned}
& s_{\chi} c=c^{2}-\mathcal{L}_{\xi} c-i_{\gamma}(A) \\
& s_{\chi} A=D c-\mathcal{L}_{\xi} A-i_{\gamma}\left(\frac{\delta \chi}{\delta A}\right)
\end{aligned}
$$

From (3.10) we conclude that the gauge-fixed BRST operator is nilpotent only up to terms proportional to the equations of motion of $A$ :

$$
s_{\chi}^{2}=-\int_{M_{3}} \operatorname{Tr}\left[i_{\gamma}\left(\frac{\delta \Gamma_{\chi}}{\delta A}\right) \frac{\delta}{\delta A}\right]
$$

Note that although the gauge-fixing part of the gauge-fixed action (3.14) is not $s_{\chi}$-exact, the change of $\Gamma_{\chi}$ under variation of $\chi$

$$
\chi \rightarrow \chi+\delta \chi
$$

is $s_{\chi}$-trivial:

$$
\delta \Gamma_{\chi}=s_{\chi}(\delta \chi[\Phi])
$$

This is so thanks to the $\chi$-dependence of the gauge-fixed BRST operator $s_{\chi}$.

Let us also observe that when $\chi[\Phi]$ does not depend on the reparametrization ghost $\xi^{\mu}$ one has

$$
s_{\chi} \chi[\Phi]=\left(S_{\chi}+\mathcal{L}_{\xi}\right) \chi[\Phi]
$$

where $S_{\chi}$ is the gauge-fixed $S$. Therefore, by taking $\chi[\Phi]$ invariant under simultaneous reparametrizations of both fields and backgrounds, one obtains the gauge-fixed BV action

$$
\Gamma_{\chi}=\Gamma_{C S}[A]+S_{\chi}(\chi[\Phi])-\frac{1}{2} \int_{M_{3}} \operatorname{Tr}\left(i_{\gamma}\left(\frac{\delta \chi}{\delta A}\right) \frac{\delta \chi}{\delta A}\right)
$$

which is independent of the reparametrization ghost $\xi^{\mu}$. Hence

$$
S_{\chi} \Gamma_{\chi}=\left(S_{0}+G_{\gamma}\right) \Gamma_{\chi}=0
$$




\subsection{A digression: Chern-Simons global vector super- symmetry}

The somewhat surprising outcome of our analysis so far is that to put CS theory consistently on a curved manifold we must couple it not only to the metric $g_{\mu \nu}$ and to its BRST partner $\psi_{\mu \nu}$ but also to the commuting vector field $\gamma^{\mu}$.

Coupling to classical background is one way to study the (quantum) properties of the corresponding conserved currents in flat space. In this subsection we make a digression from the main line of the paper and pause to understand the necessity of the source $\gamma^{\mu}$ from the point of view of the global symmetries of CS theory in flat space.

The background metric $g_{\mu \nu}$ is of course associated to the conserved symmetric stress-energy tensor $T_{\mu \nu}$. Classically, the topological nature of the theory is expressed by the relation

$$
T_{\mu \nu}=S_{0} S_{\mu \nu}
$$

where $S_{\mu \nu}$ is associated to the source $\psi^{\mu \nu}$ and is obtained from the gaugefermion functional

$$
S_{\mu \nu}=\frac{\delta \chi}{\delta g^{\mu \nu}}
$$

We would like to understand if $S_{\mu \nu}$ correspond or not to symmetries of the theory in flat space and to which current do the sources $\gamma^{\mu}$ correspond to. To answer these questions let us look at the $\gamma$-dependent part of the gauge-fixed action

$$
\begin{aligned}
G_{\gamma}(\chi[\Phi])-\frac{1}{2} \int_{M_{3}} \operatorname{Tr}\left(i_{\gamma}\left(\frac{\delta \chi}{\delta A}\right) \frac{\delta \chi}{\delta A}\right)= \\
\quad=\int_{M_{3}} \operatorname{Tr}\left[\frac{\delta \chi}{\delta c} i_{\gamma}(A)+\frac{\delta \chi}{\delta \Lambda} \mathcal{L}_{\gamma} b-\frac{1}{2} \epsilon^{\mu \nu \rho} \gamma^{\sigma} g_{\rho \sigma} \frac{\delta \chi}{\delta A_{\mu}} \frac{\delta \chi}{\delta A_{\nu}}\right]
\end{aligned}
$$

Consider for concreteness Landau's gauge

$$
\chi[\Phi]=\int_{M_{3}} \operatorname{Tr}\left[b \bar{D}^{\dagger} A\right]=\int_{M_{3}} \sqrt{g} b^{(0)} \bar{D}^{\mu} A_{\mu} d^{3} x
$$

where $\bar{D}^{\dagger}$ is the exterior differential which is Hodge-dual to

$$
\bar{D}=d x^{\mu} \bar{D}_{\mu}
$$


$\bar{D}_{\mu}$ is the derivate covariant with respect to the Levi-Civita connection built with $g_{\mu \nu}$ and $b^{(0)}$ is the 0 -form dual to $b$. For such choice one has

$$
\frac{\delta \chi}{\delta c}=\frac{\delta \chi}{\delta \Lambda}=0
$$

Thus, the $\gamma$-dependent part of the gauge-fixed action in Landau's gauge reduces to

$$
-\int_{M_{3}} \frac{1}{2} \epsilon^{\mu \nu \rho} \gamma^{\sigma} g_{\rho \sigma} \operatorname{Tr} \frac{\delta \chi}{\delta A_{\mu}} \frac{\delta \chi}{\delta A_{\nu}}=-\int_{M_{3}} \frac{1}{2} \epsilon^{\mu \nu \rho} \gamma^{\sigma} g_{\rho \sigma} \operatorname{Tr} \partial_{\mu} b^{(0)} \partial_{\nu} b^{(0)}
$$

From this we see that the gauge-fixed action in flat space becomes independent of $\gamma^{\mu}$ when $\gamma^{\mu}$ is constant. In this limit, the BRST operator

$$
G_{\gamma} \rightarrow \gamma^{\mu} G_{\mu}
$$

turns into a global vector supersymmetry which acts on the fields as follows

$$
\gamma^{\mu} G_{\mu} c=-i_{\gamma}(A) \quad \gamma^{\mu} G_{\mu} A=-i_{\gamma}\left(\bar{D}^{\dagger} b\right)
$$

This is the vector global supersymmetry of CS gauge-fixed action in Landau's gauge which was first discovered in [15] and then extensively studied, for example, in [16, 17, 18]. We see that, in a sense, such a global symmetry is the remnant, in flat space, of the equivariant part of the topological BRST symmetry on curved manifolds: it is associated to the super-ghost $\gamma^{\mu}$, which is required to render equivariant the coupling of CS to topological gravity. Our discussion characterizes the most general gauges which enjoy global vector supersymmetry: these are the gauges for which the $\gamma$-dependent terms of the action, given by Eq. (3.24), vanish in flat space. Another example, beyond the Landau's gauge, is the axial gauge [19] for which, indeed, both (3.27) and (3.28) hold 12 .

One might therefore think that $S_{\mu \nu}$ are the conserved super-currents associated to the charges $G_{\mu}$. But this is not quite true, as we will see momentarily. One can verify that the conserved super-currents $\tilde{S}_{\mu \nu}$ associated to flat space symmetries $G_{\mu}$ are not symmetric in the indices $\mu$ and $\nu$ :

$$
\begin{array}{ll}
\tilde{S}_{\mu \nu}=S_{\mu \nu}+\Delta_{\mu \nu} & \partial^{\mu} \tilde{S}_{\mu \nu}=0 \\
S_{\mu \nu}=S_{\nu \mu} & \Delta_{\mu \nu}=-\Delta_{\nu \mu}
\end{array}
$$

\footnotetext{
${ }^{12}$ For axial gauge (3.27) and (3.28) would be valid even in curved space, but the definition of the axial gauge requires the existence of a Killing vector, which restricts the possible background metrics to the one with continuous isometries. In this case too therefore the vector supersymmetry is a global one.
} 
where

$$
\Delta_{\mu \nu}=S_{0} J_{\mu \nu} \quad J_{\mu \nu}=\frac{1}{2} \epsilon_{\mu \nu \rho} \operatorname{Tr} D^{\rho} b^{(0)} b^{(0)}
$$

Neither $S_{\mu \nu}$ nor $\Delta_{\mu \nu}$ is conserved. This is consistent with conservation of the stress-energy tensor $T_{\mu \nu}$ since the latter implies conservation of $S_{\mu \nu}$ only up to $S_{0}$-trivial terms:

$$
\begin{aligned}
& T_{\mu \nu}=S_{0} \tilde{S}_{\mu \nu}=S_{0} S_{\mu \nu} \\
& 0=\partial^{\mu} T_{\mu \nu}=S_{0} \partial^{\mu} \tilde{S}_{\mu \nu}=S_{0} \partial^{\mu} S_{\mu \nu}
\end{aligned}
$$

Since the truly conserved super-currents $\tilde{S}_{\mu \nu}$ are not symmetric, it is not possible to turn the global symmetries of the theory into local ones, by only using sources $h^{\mu \nu}=g^{\mu \nu}-\delta^{\mu \nu}$ and $\psi^{\mu \nu}$ symmetric in the indices $\mu$ and $\nu$. One needs to add one more source, anti-symmetric in the indices $\mu$ and $\nu$

$$
\begin{aligned}
& h^{\mu \nu} T_{\mu \nu}+\psi^{\mu \nu} S_{\mu \nu}+\phi^{\mu \nu} S_{0} J_{\mu \nu}+\cdots \\
& \delta h^{\mu \nu}=D^{(\mu} \xi^{\nu)} \quad \delta \psi^{\mu \nu}=D^{(\mu} \gamma^{\nu)} \quad \delta \phi^{\mu \nu}=D^{[\mu} \gamma^{\nu]}
\end{aligned}
$$

Working out the Noether procedure in a way compatible with $S_{0}$-invariance one is led to the coupling

$$
D^{[\mu} \gamma^{\nu]} J_{\mu \nu}=\frac{1}{2}\left(D^{\mu} \gamma^{\rho}\right) \epsilon_{\mu \nu \rho} \operatorname{Tr} b^{(0)} D^{\nu} b^{(0)}
$$

that was found with the BV method.

In conclusion, from the point of view of the conserved currents of the theory in flat space the coupling to topological gravity encodes the relations

$$
\partial^{\mu} T_{\mu \nu}=0 \quad T_{\mu \nu}=S_{0} S_{\mu \nu} \quad \partial^{\mu} S_{\mu \nu}=-S_{0} \partial^{\mu} J_{\mu \nu}
$$

\section{A Deformation of Chern-Simons Theory}

Let us collect the fields and anti-fields of the gauge sector into a generalized form with values in the Lie algebra of $G$ :

$$
\mathcal{A} \equiv c+A+A^{*}+c^{*}
$$

The usefulness of introducing such a field with indefinite form degree in Chern-Simons theory has been pointed out in [21] and [22]. 
$\mathcal{A}$ is odd since has total fermionic degree (form+ghost number) $f=1$. As reviewed in the Appendix $\AA$ the equivariant cohomology of $s$ modulo $d$ is equivalent to the cohomology of the coboundary operator $\delta$

$$
\delta \equiv S+i_{\gamma}-d
$$

on the space of generalized forms which do not contain the ghost field $\xi^{\mu}$. Observe that Eqs. (2.17) are equivalent to

$$
\left(G_{\gamma}+i_{\gamma}\right) \mathcal{A}=0
$$

Therefore the action of $\delta$ on it coincides with that of the "naive" $\delta_{0} \equiv$ $S_{0}-d$ :

$$
\delta \mathcal{A}=\delta_{0} \mathcal{A}
$$

The BRST transformations (2.11) rewrite as follows

$$
\delta \mathcal{A}=\delta_{0} \mathcal{A}=\mathcal{A}^{2}
$$

It is well known that elements of the local $\delta_{0}$-cohomology made of $\mathcal{A}$ are built with the help of $G$-invariant anti-symmetric polynomials in the Lie algebra of $G$ [20]. If $\tau_{a_{1}, \ldots a_{m}}$ is a $G$-invariant completely anti-symmetric tensor with $m$ indices $a_{i}$ running in the adjoint of $G$, the generalized form

$$
\left\langle\mathcal{A}^{m}\right\rangle \equiv \tau_{a_{1}, \ldots a_{m}} \mathcal{A}^{a_{1}} \ldots \mathcal{A}^{a_{m}}
$$

corresponds to a non-trivial class of $\delta_{0}$. Thanks to the "chirality" property (4.3) of $\mathcal{A}$, this is also a class of the equivariant $\delta$.

The smallest value of $m$ is $m=3$, for which there is a unique choice $\tau_{a b c}=f_{a b c}$, the structure constants of the Lie algebra of $G$. One can write the corresponding observable

$$
\Omega_{3} \equiv \frac{1}{3} \operatorname{Tr} \mathcal{A}^{3} \equiv \Omega_{3}^{(0)}+\Omega_{2}^{(1)}+\Omega_{1}^{(2)}+\Omega_{0}^{(3)}
$$

in terms of traces over some irreducible representation $R$, the dependence on the representation being an overall multiplicative factor. The forms

$$
\begin{aligned}
& \Omega_{3}^{(0)}=\frac{1}{3} \operatorname{Tr} c^{3} \\
& \Omega_{2}^{(1)}=\operatorname{Tr} A c^{2} \\
& \Omega_{1}^{(2)}=\operatorname{Tr}\left[A^{*} c^{2}+A^{2} c\right] \\
& \Omega_{0}^{(3)}=\frac{1}{3} \operatorname{Tr}\left[A^{3}+3 c^{*} c^{2}+3\{A, c\} A^{*}\right]
\end{aligned}
$$


satisfy the descent equations (A.12). In particular the integral of the 3-form has ghost number 0 and is both $S$ and $S_{0}$ invariant

$$
S \int_{M_{3}} \Omega_{0}^{(3)}=S_{0} \int_{M_{3}} \Omega_{0}^{(3)}=G_{\gamma} \int_{M_{3}} \Omega_{0}^{(3)}=0
$$

This cocycle is related to the CS classical action by the equation

$$
\omega_{C S}=-\frac{1}{3 !} \operatorname{Tr} \mathcal{A}^{3}+\delta_{0} \alpha
$$

where $\omega_{C S}$ is the $\delta_{0}$-cocycle

$$
\omega_{C S} \equiv \omega_{C S}^{(0)}+\omega_{C S}^{(1)}+\omega_{C S}^{(2)}+\omega_{C S}^{(3)}
$$

whose 3-form component is the CS form

$$
\begin{aligned}
& \omega_{C S}^{(3)}=\operatorname{Tr}\left[\frac{1}{2} A d A+\frac{1}{3} A^{3}\right]=-\frac{1}{2 !}\left[\Omega_{0}^{(3)}+d \operatorname{Tr} A^{*} c-S_{0} \operatorname{Tr}\left[A^{*} A+c^{*} c\right]\right] \\
& \omega_{C S}^{(2)}=\frac{1}{2 !} d A c=-\frac{1}{2 !}\left[\Omega_{1}^{(2)}-S_{0} \operatorname{Tr} A^{*} c\right] \\
& \omega_{C S}^{(1)}=-\frac{1}{2 !} \Omega_{2}^{(1)} \\
& \omega_{C S}^{(0)}=-\frac{1}{2 !} \Omega_{3}^{(0)}
\end{aligned}
$$

and $\alpha$ is a generalized form of total ghost number $f=2$ :

$$
\alpha=\frac{1}{2 !} \operatorname{Tr}\left[A^{*} c+A^{*} A+c^{*} c\right]
$$

Thus $\Omega_{0}^{(3)}$ and the CS classical form $\omega_{C S}^{(3)}$ are equivalent (up to a multiplicative factor) in the "naive" $S_{0}$ cohomology (modulo d). However, $\alpha$ does not satisfy the "chirality" condition (4.3). Therefore $\omega_{C S}^{(3)}$ is not an equivariant cocycle, and it is not equivalent to $\Omega_{0}^{(3)}$ in the equivariant $s$-cohomology (modulo d). As we verified in Section 3, to construct an equivariant action starting from the classical CS form, one must introduce explicit couplings to the topological gravity fields.

Since $\Omega_{0}^{(3)}$ is $S$-invariant modulo $d$ we can add it to the BV action

$$
\tilde{\Gamma}_{B V}(t)=\Gamma_{B V}+t \int_{M_{3}} \Omega_{0}^{(3)}
$$


The new BV action generates deformed BRST transformation rules. It is straightforward to verify that the new action is just the rescaled original BV action

$$
\tilde{\Gamma}_{B V}(t)=\left.\frac{1}{(1+t)^{2}} \Gamma_{B V}(\mathcal{A})\right|_{\mathcal{A} \rightarrow(1+t) \mathcal{A}}
$$

In this sense, the effect of adding this observable to the BV action is just to renormalize both the coupling constant and the fields.

Following the suggestion of [4] we will now consider cocycles with $m>3$. The number and structure of the invariant tensors $\tau_{a_{1}, \ldots, a_{m}}$ with $m>3$ depend on the gauge group $G$. In the rest of this Section we will restrict our discussion to $G=S U(N)$, both for concreteness and because we have in mind an application to topological D-branes.

A special class of $S U(N)$-invariant tensors are anti-symmetrized single traces of an odd number of $S U(N)$ generators in some irreducible representation $R$

$$
\tau_{a_{1}, \ldots a_{m}}^{(R)}=\operatorname{Tr}_{R} T^{\left[a_{1}\right.} \cdots T^{\left.a_{m}\right]}
$$

If $m$ takes one of the following $N-1$ values

$$
m=3,5,7, \ldots, 2 N-1
$$

the dependence of $\tau_{a_{1}, \ldots a_{m}}^{(R)}$ on the representation $R$ is a multiplicative possibly vanishing — normalization factor 13 :

$$
\tau_{a_{1}, \ldots a_{m}}^{(R)}=d_{m}(R) \tau_{a_{1}, \ldots a_{m}}
$$

with $\tau_{a_{1}, \ldots a_{m}}$ independent of $R$.

All the other $S U(N)$ anti-symmetric tensors are multi-traces. They are obtained by multiplying and anti-symmetrizing single trace invariants with $m$ in the range (4.17). In the following we will therefore restrict ourselves to such "primitive" single trace invariants.

The observables corresponding to the single trace invariants are

$$
\Omega_{m}=\frac{1}{m} \operatorname{Tr} \mathcal{A}^{m} \equiv \Omega_{m}^{(0)}+\Omega_{m-1}^{(1)}+\Omega_{m-2}^{(2)}+\Omega_{m-3}^{(3)}
$$

where

$$
\Omega_{m}^{(0)}=\frac{1}{m} \operatorname{Tr} c^{m}
$$

\footnotetext{
${ }^{13}$ The factor $d_{m}(R)$ vanishes for $R=\bar{R}$ and $m=5,9,11, \ldots$
} 


$$
\begin{aligned}
\Omega_{m-1}^{(1)}= & \operatorname{Tr} A c^{m-1} \\
\Omega_{m-2}^{(2)}= & \operatorname{Tr}\left[A^{*} c^{m-1}+\frac{1}{2}\left(A^{2} c^{m-2}+A c A c^{m-3}+\cdots+A c^{m-2} A\right)\right] \\
\Omega_{m-3}^{(3)}=\operatorname{Tr} & {\left[c^{*} c^{m-1}+\frac{1}{2} A^{*}\left(A c^{m-2}+c A c^{m-3}+\cdots+c^{m-2} A\right)+\right.} \\
& \left.+\sum_{i, j \leq m-3} A c^{m-i} A c^{j} A c^{i-j-3}\right]
\end{aligned}
$$

The 3-forms $\Omega_{m-3}^{(3)}$ have ghost number $m-3$. By adding them to the original BV action one obtains the generic single trace deformation of $S U(N)$ CS theory

$$
\tilde{\Gamma}_{B V}\left(t_{i}\right)=\Gamma_{B V}+\sum_{i=1}^{N-1} t_{i} \int_{M_{3}} \Omega_{2 i}^{(3)}
$$

The new BV action generates deformed nilpotent BRST transformation rules which can be recast as follows

$$
\begin{aligned}
& \delta_{t} \mathcal{A}=\left\{\tilde{\Gamma}_{B V}\left(t_{i}\right), \mathcal{A}\right\}=\mathcal{A}^{2}+\sum_{i=1}^{N-1} t_{i} \mathcal{A}^{2 i+2} \\
& \delta_{t}^{2}=0
\end{aligned}
$$

where

$$
\left(\mathcal{A}^{m-1}\right)^{a} \equiv g^{a a_{1}} \tau_{a_{1} a_{2} \ldots a_{m}} \mathcal{A}^{a_{2}} \cdots \mathcal{A}^{a_{m}}
$$

and $g^{a b}$ is the invariant Killing metric on the Lie algebra of $G$.

\section{Effective Action}

Quantum averages of matter observables are formally obtained by integrating the gauge-fixed BV path integral over the fields of the gauge sector

$$
\mathrm{e}^{i \frac{k}{2 \pi} F\left[g_{\mu \nu}, \psi_{\mu \nu}, \gamma^{\mu} ; t_{i}\right]}=\int[d A d c d b d \Lambda] \mathrm{e}^{\left.i \frac{k}{2 \pi} \tilde{\Gamma}_{B V}\left(t_{i}\right)\right|_{\Phi^{*}}=\frac{\delta \chi}{\partial \Phi}}
$$

The classical Ward identities for the effective action $F[g, \psi, \gamma ; t]$ read as follows

$$
S F\left[g_{\mu \nu}, \psi_{\mu \nu}, \gamma^{\mu} ; t_{i}\right]=\int_{M_{3}}\left[\frac{\delta F}{\delta g_{\mu \nu}} \psi_{\mu \nu}-\frac{\delta F}{\delta \psi_{\mu \nu}} \mathcal{L}_{\gamma} g_{\mu \nu}\right]=0
$$


Let us consider $G=S U(N)$. Conservation of total ghost number implies

$$
F\left[g_{\mu \nu}, \psi_{\mu \nu}, \gamma^{\mu} ; t_{i}\right]=\sum_{n=0}^{\infty} F_{2 n}\left[g_{\mu \nu}, \psi_{\mu \nu}, \gamma^{\mu} ; t_{i}\right]
$$

where $F_{2 n}\left[g_{\mu \nu}, \psi_{\mu \nu}, \gamma^{\mu} ; t_{i}\right]$ is a functional of the topological gravity fields of ghost number $2 n$ which is polynomial in the couplings $t_{i}$ :

$$
F_{2 n}\left[g_{\mu \nu}, \psi_{\mu \nu}, \gamma^{\mu} ; t_{i}\right]=\sum_{\sum_{\alpha} i_{\alpha}=n} \mathcal{F}_{i_{1} ; i_{2} ; \ldots ; i_{\alpha} ; \ldots}\left[g_{\mu \nu}, \psi_{\mu \nu}, \gamma^{\mu}\right] t_{i_{1}} \cdots t_{i_{\alpha}} \cdots
$$

In other words the observables that contribute to the effective action of fixed ghost number $2 n$ are those which satisfy the selection rule

$$
\sum_{\alpha} i_{\alpha}=n \quad 1 \leq i_{\alpha} \leq N-1
$$

Therefore for finite $N$ there is only a finite number of terms in the sums (5.3) and (5.4).

Let us recall [10, 7] the geometrical interpretation of the classical Ward identity (5.2). Let $\mathrm{Met}_{3}$ be the space of 3-dimensional metrics on $M_{3}$, $\operatorname{Diff}\left(M_{3}\right)$, the diffeomorphisms of $M_{3}$ and

$$
\mathcal{M}=\operatorname{Met}_{3} / \operatorname{Diff}\left(M_{3}\right)
$$

the - possibly infinite-dimensional — associated orbit space. We will refer to it as the moduli space of 3-dimensional metrics.

Let $m \equiv\left\{m^{a}\right\}$ be local coordinates on $\mathcal{M}$ and $\bar{g}_{\mu \nu}(x ; m)$ a local section of the bundle $\mathcal{B}$ whose total space is $M_{e t}$ and whose base is $\mathcal{M}$. Let

$$
d_{m} \equiv d m^{a} \frac{\partial}{\partial m^{a}}
$$

the exterior derivative on $\mathcal{M}$. When acting on $\bar{g}_{\mu \nu}(x ; m), d_{m}$ does not in general produce a tensor covariant under $m$-dependent diffeomorphisms of $M_{3}$. One has

$$
d_{m} \bar{g}_{\mu \nu}(x, m)=\bar{\psi}_{\mu \nu}(x ; m)-\mathcal{L}_{\bar{\xi}} \bar{g}_{\mu \nu}(x ; m)
$$

where $\bar{\psi}_{\mu \nu}(x, m)$ is covariant under $m$-dependent $M_{3}$-diffeomorphisms - i.e. it is a local section of the cotangent bundle $T^{*} \mathcal{M}$ - and $\bar{\xi}^{\mu}(x ; m)$ is an $m$ dependent vector field on $M_{3}$ with values in the local 1-forms on $\mathcal{M} . \bar{\xi}^{\mu}(x ; m)$ defines a local connection on the bundle $\mathcal{B}$. 
Nilpotency of $d_{m}$ implies

$$
d_{m} \bar{\psi}_{\mu \nu}(x, m)=\mathcal{L}_{\bar{\gamma}} \bar{g}_{\mu \nu}(x ; m)-\mathcal{L}_{\bar{\xi}} \bar{\psi}_{\mu \nu}(x ; m)
$$

where $\bar{\gamma}^{\mu}(x ; m)$ is a $m$-dependent vector field on $M_{3}$ with values in the 2 forms on $\mathcal{M}$. $\bar{\gamma}^{\mu}(x ; m)$ is related to the curvature 2-form of the connection $\bar{\xi}^{\mu}(x ; m)$

$$
d_{m} \bar{\xi}^{\mu}(x ; m)+\frac{1}{2} \mathcal{L}_{\bar{\xi}} \bar{\xi}^{\mu}(x ; m)=\bar{\gamma}^{\mu}(x ; m)
$$

Thus the action of $d_{m}$ on the local sections $\bar{g}_{\mu \nu}(x ; m), \bar{\psi}_{\mu \nu}(x ; m)$ and $\bar{\gamma}^{\mu}(x ; m)$ is identical to the action (2.8) of the equivariant BRST operator $s$ on the topological gravity fields.

In conclusion, the classical Ward identity (5.2) states that the effective action $F_{2 n}$, when evaluated on the backgrounds $\bar{g}_{\mu \nu}(x ; m), \bar{\psi}_{\mu \nu}(x ; m)$ and $\bar{\gamma}^{\mu}(x ; m)$, gives rise to a local closed $2 n$-form $\bar{F}_{2 n}$ on moduli space $\mathcal{M}$.

The equivariance condition ensures that the local form $\bar{F}_{2 n}$ is actually globally defined on $\mathcal{M}$. In fact, equivariance means that $\bar{F}_{2 n}$ is independent of the choice of the connection $\bar{\xi}^{\mu}(x ; m)$ which had to be introduced in order to define $\bar{\psi}_{\mu \nu}(x ; m)$ and $\bar{\gamma}^{\mu}(x ; m)$. Since $\bar{\psi}_{\mu \nu}(x ; m)$ and $\bar{\gamma}^{\mu}(x ; m)$ transform covariantly under $m$-dependent diffeomorphisms - unlike $\bar{\xi}^{\mu}(x ; m)$ - the local form $\bar{F}_{2 n}$ extends to a globally defined form on $\mathcal{M}$.

As explained in [4], it makes sense, in this situation, to integrate the closed, globally defined form $\bar{F}_{2 n}$ on $2 n$-dimensional cycles $C_{2 n}$ of moduli space $\mathcal{M}$

$$
I_{2 n}\left(t_{i}\right)=\int_{C_{2 n}} F_{2 n}\left[\bar{g}_{\mu \nu}(x ; m), \bar{\psi}_{\mu \nu}(x ; m), \bar{\gamma}^{\mu}(x ; m) ; t_{i}\right]
$$

Were the classical Ward identity satisfied, the $I_{2 n}\left(t_{i}\right)$ 's would be topological invariants of the 3 -dimensional manifold $M_{3}$ depending on the homology class of $C_{2 n}$. In the next Section we will consider the possible quantum anomalies that can appear on the right-hand side of the classical Ward identity (5.2).

\section{Topological Anomalies}

Anomalous Ward identities for the $G=S U(N)$ theory

$$
S F_{2 n}[g, \psi, \gamma]=\int_{M_{3}} A_{2 n+1}^{(3)}[g, \psi, \gamma]
$$


involve local functionals of the topological gravity fields of ghost number $2 n+1$ which are $S$-closed modulo $d$ :

$$
S A_{2 n+1}^{(3)}=d A_{2 n+2}^{(2)}
$$

$A_{2 n+2}^{(2)}$ is a 2 -form of ghost number $2 n+2$. This implies that $A_{2 n+1}^{(3)}$ is the 3 -form component of a generalized anomaly form

$$
\mathcal{A}_{2 n+4}=A_{2 n+1}^{(3)}+A_{2 n+2}^{(2)}+A_{2 n+3}^{(1)}+A_{2 n+4}^{(0)}
$$

which is $\delta$-closed

$$
\delta \mathcal{A}_{2 n+4}=0
$$

Local solutions of (6.4) modulo $\delta$ are local observables of 3-dimensional topological gravity. Construction of topological gravity observables in various dimensions has been discussed by several authors, starting from [23, 24]. Although the formalism developed in [23] applies to any dimension, that work and most of the subsequent ones focused mainly on either 2 or (less so) 4 dimensions. In particular, as far as we know, no explicit formulae have been exhibited in dimension 3 . We think therefore it is appropriate to present here a review of the relevant result:14

To find local solutions of Eq. (6.4) we start from the matrix-valued curvature 2 -form

$$
\left(R^{(2)}\right)^{\mu}{ }_{\nu}=\frac{1}{2}\left(R_{\alpha \beta}\right)_{\nu}^{\mu} d x^{\alpha} d x^{\beta}=\left(d \Gamma+\Gamma^{2}\right)^{\mu}{ }_{\nu}
$$

where $\Gamma$ is the matrix-valued 1 -form

$$
(\Gamma)_{\nu}^{\mu}=\Gamma_{\alpha \nu}^{\mu} d x^{\alpha}
$$

From

$$
\mathcal{L}_{\gamma} \Gamma=D R^{(0)}-i_{\gamma} R^{(2)}
$$

one derives the following matrix-valued descent equations:

$$
\begin{aligned}
& S R^{(2)}=D R^{(1)} \\
& S R^{(1)}=D R^{(0)}-i_{\gamma} R^{(2)} \\
& S R^{(0)}=-i_{\gamma} R^{(1)}
\end{aligned}
$$

\footnotetext{
${ }^{14}$ We believe that our discussion is slightly more general than the original one [23], since we explicitly show in the Appendix B that the trace classes we consider do not depend on the choice of the particular curvature two-form, be it with or without "torsion".
} 
where we introduced the matrix-valued 1 and 0 -forms

$$
\begin{aligned}
\left(R^{(1)}\right)_{\nu}^{\mu} & \equiv S \Gamma_{\nu}^{\mu}=\frac{1}{2}\left[D \psi_{\nu}^{\mu}+D_{\nu} \psi_{\alpha}^{\mu} d x^{\alpha}-D^{\mu} \psi_{\alpha \nu} d x^{\alpha}\right] \\
\left(R^{(0)}\right)_{\nu}^{\mu} & \equiv D_{\nu} \gamma^{\mu}
\end{aligned}
$$

Let us define the matrix-valued generalized form of total fermionic degree 2

$$
\mathcal{R} \equiv R^{(2)}+R^{(1)}+R^{(0)}
$$

and the differential

$$
\delta \equiv S+i_{\gamma}-D
$$

acting on matrix-valued generalized forms $X$. It easy to see that

$$
\delta^{2} X=[\mathcal{R}, X]
$$

Hence $\delta$ is nilpotent when acting on reparametrization scalars. Moreover one has

$$
\delta \mathcal{R}=0
$$

which is equivalent to Eqs. (6.8). The invariants constructed with $\mathcal{R}$

$$
\mathcal{A}_{2 k}=\operatorname{tr} \mathcal{R}^{k} \quad k=1,2, \ldots
$$

where the trace is taken on the matrix Lorentz indices, are therefore $\delta$ cocycles of total ghost number $2 k$ :

$$
\delta \mathcal{A}_{2 k}=0
$$

Let us discuss if they are trivial or not. To this end, consider the decomposition of the matrix $\mathcal{R}$

$$
\mathcal{R} \equiv \tilde{\mathcal{R}}+\tilde{\mathcal{F}}
$$

into its symmetric and antisymmetric parts, with respect to $g^{\mu \nu}$ :

$$
\begin{aligned}
& (\tilde{\mathcal{R}})^{\mu}{ }_{\nu} g^{\nu \lambda}=-g^{\mu \nu}(\tilde{\mathcal{R}})^{\lambda}{ }_{\nu} \\
& (\tilde{\mathcal{F}})^{\mu}{ }_{\nu} g^{\nu \lambda}=g^{\mu \nu}(\tilde{\mathcal{F}})_{\nu}^{\lambda}
\end{aligned}
$$

Explicitly:

$$
\begin{aligned}
\left(\tilde{R}^{(2)}\right)_{\nu}^{\mu} & =\left(R^{(2)}\right)_{\nu}^{\mu} \\
\left(\tilde{R}^{(1)}\right)_{\nu}^{\mu} & =\frac{1}{2}\left[D_{\nu} \psi_{\alpha}^{\mu} d x^{\alpha}-D^{\mu} \psi_{\alpha \nu} d x^{\alpha}\right] \\
\left(\tilde{R}^{(0)}\right)_{\nu}^{\mu} & =\frac{1}{2}\left(D_{\nu} \gamma^{\mu}-D^{\mu} \gamma_{\nu}\right)
\end{aligned}
$$


and

$$
\begin{aligned}
\left(\tilde{\mathcal{F}}^{(2)}\right)^{\mu}{ }_{\nu} & =0 \\
\left(\tilde{\mathcal{F}}^{(1)}\right)^{\mu}{ }_{\nu} & =\frac{1}{2} D \psi_{\nu}^{\mu} \\
\left(\tilde{\mathcal{F}}^{(0)}\right)^{\mu}{ }_{\nu} & =\frac{1}{2}\left(D_{\nu} \gamma^{\mu}+D^{\mu} \gamma_{\nu}\right)
\end{aligned}
$$

The important observation is

$$
\begin{aligned}
\tilde{\mathcal{F}}_{\nu}^{\mu} & =-\frac{1}{2} g^{\mu \lambda} \delta \psi_{\nu \lambda}=-\delta \mathcal{C}_{\nu}^{\mu}+\frac{1}{2} \delta\left(g^{\mu \lambda}\right) \psi_{\nu \lambda}= \\
& =-\delta \mathcal{C}_{\nu}^{\mu}-\frac{1}{2} \psi^{\mu \lambda} \psi_{\nu \lambda}=-\left(\delta \mathcal{C}+\mathcal{C}^{2}\right)_{\nu}^{\mu}-\frac{1}{4} \psi^{\mu \lambda} \psi_{\nu \lambda}
\end{aligned}
$$

where $\mathcal{C}$ is the matrix of fermionic number +1 :

$$
\mathcal{C}_{\nu}^{\mu} \equiv \frac{1}{2} \psi_{\nu}^{\mu}
$$

The decomposition (6.16) rewrites therefore as follows

$$
\mathcal{R}=\hat{\mathcal{R}}-\mathcal{F}
$$

where

$$
\hat{\mathcal{R}} \equiv \tilde{\mathcal{R}}-\frac{1}{4} \psi^{\mu \lambda} \psi_{\nu \lambda} \quad \mathcal{F} \equiv \delta \mathcal{C}+\mathcal{C}^{2}
$$

Since $\psi_{\nu}^{\mu}$ is anti-commuting, $\hat{\mathcal{R}}$ is anti-symmetric with respect to $g^{\mu \nu}$ :

$$
(\hat{\mathcal{R}})^{\mu}{ }_{\nu} g^{\nu \lambda}=-g^{\mu \nu}(\hat{\mathcal{R}})^{\lambda}{ }_{\nu}
$$

It should be noted, however, that, $\mathcal{F}$ is not symmetric with respect to $g^{\mu \nu}$.

Since

$$
\delta \mathcal{F}=\delta^{2} \mathcal{C}+[\delta \mathcal{C}, \mathcal{C}]=[\hat{\mathcal{R}}, \mathcal{C}]
$$

one has

$$
\delta \hat{\mathcal{R}}=-[\mathcal{C}, \hat{\mathcal{R}}]
$$

It is therefore convenient to introduce a new differential $\hat{\delta}$ whose action on generalized matrix-valued forms $X$ is

$$
\hat{\delta} X \equiv \delta X+[\mathcal{C}, X]
$$


The curvature of $\hat{\delta}$ is $\hat{\mathcal{R}}$

$$
\hat{\delta}^{2} X=[\hat{\mathcal{R}}, X]
$$

Eq. (6.26) becomes the Bianchi identity for $\hat{\mathcal{R}}$ :

$$
\hat{\delta} \hat{\mathcal{R}}=0
$$

and Eq. (6.25) rewrites

$$
\hat{\delta} \mathcal{F}=[\mathcal{R}, \mathcal{C}]
$$

Hence the invariants constructed with $\hat{\mathcal{R}}$

$$
\hat{\mathcal{A}}_{2 k}=\operatorname{tr} \hat{\mathcal{R}}^{k}
$$

are $\delta$-closed:

$$
\delta \hat{\mathcal{A}}_{2 k}=0
$$

Comparison with (6.14) gives

$$
\delta\left(\hat{\mathcal{A}}_{2 k}-\mathcal{A}_{2 k}\right)=0
$$

One can check that the differences

$$
\mathcal{B}_{2 k} \equiv \hat{\mathcal{A}}_{2 k}-\mathcal{A}_{2 k}=\operatorname{tr} \mathcal{F}^{k}+k \operatorname{tr} \mathcal{F}^{k-1} \mathcal{R}+\cdots
$$

are trivial cocycles and thus

$$
\mathcal{A}_{2 k} \equiv \hat{\mathcal{A}}_{2 k} \quad \text { in } \delta \text {-cohomology }
$$

We show this, for any space-time dimensions, in the Appendix B, In the Appendix B we show also a more general proposition: The non-vanishing cohomology of $\delta$ on the space of invariant polynomials built with the matrices $\hat{\mathcal{R}}, g^{\mu \nu}$ and $\mathcal{F}$ is generated by the invariant polynomials built with of $\hat{\mathcal{R}}$ and $g^{\mu \nu}$. The trace classes $\mathcal{A}_{2 k}$ do not involve explicitly the metric, but only the curvature forms. Therefore these classes can be equivalently written in terms of either the $\mathcal{R}$ or the $\hat{\mathcal{R}}$ curvature form 15 .

\footnotetext{
${ }^{15}$ The original works $[23,24]$ studying topological gravity observables focused on even dimensions 2 and 4 . In even dimensions one can consider, beyond the trace classes, the Euler class. This class involves the metric and therefore it is more conveniently written in terms of $\hat{\mathcal{R}}$ and $g^{\mu \nu}$ : It necessarily contains $\mathcal{F}$ when expressed in terms of the full curvature form $\mathcal{R}$. For this reason the authors of 23, 24, chose a formalism in which $\hat{\mathcal{R}}$ and $g^{\mu \nu}$, but not $\mathcal{R}$, appear.
} 
Since $\hat{\mathcal{R}}$ is anti-symmetric with respect to $g^{\mu \nu}$ the traces of the odd powers of $\hat{\mathcal{R}}$ vanish identically. In conclusion, in generic dimensions, we end up with the following non-trivial equivariant cocycles

$$
\mathcal{A}_{4 p} \equiv \hat{\mathcal{A}}_{4 p} \equiv \operatorname{tr} \mathcal{R}^{2 p} \quad p=1,2, \ldots
$$

Let us now specialize this discussion to the case at hand, i.e. to dimension 3. In such dimension traces of powers of $\hat{\mathcal{R}}$ are all proportional to powers of $\operatorname{tr} \hat{\mathcal{R}}^{2}$. Hence there exists one single non-trivial cocycle for any given total fermionic degree $4 p$

$$
\mathcal{A}_{4 p} \sim \mathcal{A}_{4}^{p} \equiv\left(\operatorname{tr} \mathcal{R}^{2}\right)^{p}
$$

The corresponding CS topological anomaly has ghost number $4 p-3$ :

$$
S F_{4(p-1)}[g, \psi, \gamma]=c_{2(p-1)}(t) \int_{M_{3}}\left(\operatorname{tr} \mathcal{R}^{2}\right)^{p} \quad p=1,2, \ldots
$$

where the integration over $M_{3}$ selects the component of the anomaly of form degree 3 . For $p=1$ we obtain the well-known framing anomaly of CS theory [2, 3]:

$$
A_{1}^{(3)}=\operatorname{tr} R^{(2)} R^{(1)}=-\epsilon^{\mu \nu \rho} R_{\rho}^{\sigma} D_{\mu} \psi_{\nu \sigma} d^{3} x
$$

The anomalies with $p>1$ involve the super-ghost $\gamma^{\mu}$. They are relevant for the higher-ghost deformations of CS theory discussed in Section 4 .

The cohomological analysis does not determine, of course, the coefficient of the possible anomalies. It is known 2 that the coefficient $c_{0}$ of the anomaly for the ghost number zero effective action $F_{0}$ does not vanish. For $G=$ $S U(N)$ at 1-loop

$$
c_{0}^{1-\text { loop }}=\frac{1}{12} \frac{N^{2}-1}{k}
$$

where $\frac{1}{k}$ is the CS coupling constant. Witten [2] proposed an exact formula for $c_{0}$ motivated by the Hamiltonian solution of the theory in terms of CFT:

$$
c_{0}=\frac{1}{12} \frac{N^{2}-1}{k+N}
$$

This formula has been verified at 2-loops by an explicit perturbative computation [21]. 
The coefficients $c_{2(p-1)}$ of the anomaly of $F_{4(p-1)}$ for $p>1$ are homogeneous polynomials of degree $2(p-1)$ in the $t_{i}$ 's, with $t_{i}$ of weight $i$ :

$$
\begin{aligned}
& c_{2}\left(t_{1}, t_{2}\right)=c_{2}^{(1)} t_{2}+c_{2}^{(2)} t_{1}^{2} \\
& c_{4}\left(t_{1}, t_{2}, t_{3}, t_{4}\right)=c_{4}^{(1)} t_{4}+c_{4}^{(2)} t_{2}^{2}+c_{4}^{(3)} t_{2} t_{1}^{2}+c_{4}^{(4)} t_{3} t_{1}+c_{4}^{(5)} t_{1}^{4} \\
& \ldots \quad \ldots
\end{aligned}
$$

Thus, the polynomials $I_{2 n}\left(t_{i}\right)$ defined in Eq. (5.11) are genuine 3-dimensional topological invariants for any $n$ odd. Computation of the anomaly coefficients $c_{2(p-1)}(t)$ for $p>1$ appears to be an interesting open problem. Since $c_{2(p-1)}(t)$ with $p>1$ receives contributions from more than one higherghost matter observable, $I_{2 n}\left(t_{i}\right)$ are genuine topological invariants for $n$ even as well when restricted on some suitable non-trivial sub-manifold of $t_{i}$-space.

Let us conclude this Section with the following remark. The framing anomaly is locally trivial:

$$
A_{1}^{(3)}=S \Omega_{0}^{(3)}(\Gamma)+d \Omega_{1}^{(2)}(\Gamma)
$$

where

$$
\Omega_{0}^{(3)}(\Gamma) \equiv \frac{1}{2} \operatorname{tr} \Gamma d \Gamma+\frac{1}{3} \operatorname{tr} \Gamma^{3}
$$

is the gravitational CS action and

$$
\Omega_{1}^{(2)} \equiv \frac{1}{2} \operatorname{tr} \Gamma R^{(1)}
$$

This is best understood by considering the generalized form $\mathcal{A}_{4}$ in arbitrary dimension (i.e. different than 3 ). Its component with highest form degree is a 4 -form

$$
\left(\mathcal{A}_{4}\right)^{(4)}=A_{0}^{(4)}=\frac{1}{2} \operatorname{tr}\left(R^{(2)}\right)^{2}
$$

which is $d$-closed, by virtue of the Bianchi identity, and hence locally $d$-exact

$$
A_{0}^{(4)}=d \Omega_{0}^{(3)}(\Gamma)
$$

Therefore

$$
S \frac{1}{2} \operatorname{tr}\left(R^{(2)}\right)^{2}=d S \Omega_{0}^{(3)}(\Gamma)=d A_{1}^{(3)}
$$

which implies the local triviality relation (6.43). 
The gravitational CS action, however, is not a form and cannot be integrated on a non-trivial manifold $M_{3}$. Thus the anomaly cannot be removed, since it is not the variation of a genuine 3 -form. An alternative way to state this is to remark that the generalized anomaly form $\mathcal{A}_{4}$ is not $\delta$-exact, even locally. Indeed, by defining the gravitational CS generalized form of total degree 3

$$
\begin{aligned}
& \Omega_{3}(\Gamma) \equiv \Omega_{0}^{(3)}+\Omega_{1}^{(2)}+\Omega_{2}^{(1)} \\
& \Omega_{2}^{(1)} \equiv \frac{1}{2} \operatorname{tr} \Gamma R^{(0)}
\end{aligned}
$$

one can rewrite relations (6.47) and (6.43) as follows

$$
\mathcal{A}_{4}=\delta \Omega_{3}(\Gamma)+\omega_{4}
$$

$\omega_{4}$ is the generalized form of total fermionic degree 4

$$
\omega_{4}=\frac{1}{2} \operatorname{tr}\left[\partial \gamma\left(d \Gamma+R^{(1)}+R^{(0)}\right)\right]
$$

where $\partial \gamma$ is the matrix $\partial_{\mu} \gamma^{\nu}$. The component of form degree 3 of $\omega_{4}$ vanishes, but the components of lower degree do not. Thus, although the 3 -form anomaly is locally exact, the rest of the anomaly multiplet is not exact, even locally.

Note that Eq. (6.50) is consistent with both $\delta$-closeness of $\mathcal{A}_{4}$ and a non-vanishing $\omega_{4}$

$$
\delta \mathcal{A}_{4}=0=\delta^{2} \Omega_{3}(\Gamma)+\delta \omega_{4}
$$

since $\delta$ is nilpotent only on forms

$$
\delta^{2}=\mathcal{L}_{\gamma}-\left\{i_{\gamma}, d\right\}
$$

and $\Omega_{3}(\Gamma)$ is not a form.

\section{Topological Anomaly Inflow}

In this Section we discuss possible implications of CS topological anomalies of higher ghost number for topological strings.

CS theory with $G=S U(N)$ on a 3-manifold $M_{3}$ describes a stack of $N$ topological D-branes — of the A-type — propagating on the non-compact 
CY 6-manifold $X_{6}=T^{*} M_{3}[4]$. Such an open string interpretation of CS theory led Witten to suggest that the framing anomaly be cancelled by couplings coming from the closed string sector. Evidence for the existence of closed string couplings of this kind of (more or less) the right magnitude was presented in [26]. In this Section we will try to extend these considerations to the higher-ghost deformation of $S U(N)$ CS theory that was discussed in Section 4. We will work out the general form of the anomalous couplings of the topological closed string field theory which cancel all the higher ghost CS topological anomalies (6.38).

The quantum properties of the target space field theory describing closed topological strings of the A-type are poorly understood, although a proposal for the classical theory has been put forward in [25]. The physical vertex operators of the closed A topological model are de Rahm cohomology classes of forms on $X_{6}$ of degree 2. Consequently it is natural to assume that the corresponding target space theory contains a 2-form field $k^{(2)}$ whose linearized equations of motion read

$$
d k^{(2)}=0
$$

and whose gauge properties are described by a BRST operator $S_{0}$

$$
S_{0} k^{(2)}=d k^{(1)} \quad S_{0} k^{(1)}=d k^{(0)} \quad S_{0} k^{(0)}=0
$$

Repeating the arguments that we applied to CS theory, one arrives to the conclusion that the closed target space theory must be coupled to topological 6-dimensional gravity by introducing the equivariant BRST operator

$$
s=S-\mathcal{L}_{\xi}
$$

where

$$
S k^{(2)}=d k^{(1)} \quad S k^{(1)}=d k^{(0)}-i_{\gamma}\left(k^{(2)}\right) \quad S k^{(0)}=-i_{\gamma}\left(k^{(1)}\right)
$$

and $\gamma^{\mu}$ is the 6-dimensional reparametrizations super-ghost. However, $s$ so defined is nilpotent only up to the equations of motion of $k^{(2)}$ :

$$
S^{2}=\mathcal{L}_{\gamma}+i_{\gamma}\left(d k^{(2)}\right) \frac{\delta}{\delta k^{(2)}}
$$

We already know a way to fix this: Introduce form fields of higher degrees and define the generalized form

$$
\mathcal{K}=k^{(0)}+k^{(1)}+k^{(2)}+k^{(3)}+k^{(4)}+k^{(5)}+k^{(6)}
$$


The BRST transformations of the closed string fields $k^{(p)}$ are captured by a coboundary operator $\delta$

$$
\delta \mathcal{K} \equiv\left(S-d+i_{\gamma}\right) \mathcal{K}=0
$$

which is nilpotent off-shell

$$
\delta^{2}=0
$$

The proposal in [25] for the target space theory of A-type topological strings, advocates in fact, partly because of the CS analogy, the introduction of string fields $k^{(p)}$ with all $p$. More precisely, forms $k^{(p)}$ with $p=0,1,2$ are identified in [25] with fields while those with $p=3,4,5$ are interpreted as anti-fields. There is a further constraint on the theory which leads to vanishing 6 -form field $k^{(6)}$. As we elaborate in the following, topological anomalies considerations represent an independent reasoning that supports such a setting for the closed target space theory.

Topological $D$-branes should act as sources for the closed string fields. We expect therefore that in presence of branes wrapped around a (Lagrangian) 3-cycle $M_{3}$ of $X_{6}$ the linearized equations of motion of the closed string field (7.1) acquire a source term

$$
d k^{(2)}=\alpha \delta_{M_{3}}
$$

where $\alpha$ is a constant proportional to the D-brane charge and $\delta_{M_{3}}$, the Poincaré dual of the cycle $M_{3}$, is a closed 3-form with support on the brane. Correspondingly, the BRST transformations (7.6) should be modified, in presence of branes, as follows

$$
\delta \mathcal{K}=\alpha \delta_{M_{3}}
$$

Consistency requires

$$
\delta^{2} \mathcal{K}=\alpha \delta \delta_{M_{3}}=0
$$

which is satisfied since

$$
d \delta_{M_{3}}=0 \quad i_{\gamma}\left(\delta_{M_{3}}\right)=0 \quad S \delta_{M_{3}}=0
$$

The first of these conditions is the closeness of the Poincaré dual of the cycle $M_{3}$. The second equation is the statement that, in presence of branes, the diffeomorphisms which represent genuine gauge symmetries of the theory are those leaving $M_{3}$ invariant. Finally, the last equation is the condition that 
$M_{3}$ be a supersymmetric cycle, which, for the A-model, is the requirement that $M_{3}$ be a Lagrangian sub-manifold of $X_{6}$ [4].

In this setting there is a natural and simple way to introduce the couplings of the closed string theory which cancel the topological anomalies of the Dbrane theory. Each CS anomaly with coefficient $c_{2(p-1)}\left(t_{i}\right)$ is cancelled by a term in the closed string field action which couples the closed string field to the topological gravity observable $\mathcal{A}_{4 p}$ :

$$
I_{p}=-\frac{c_{2(p-1)}(t)}{\alpha} \int_{X_{6}} \mathcal{A}_{4 p} \mathcal{K}=-\frac{c_{2(p-1)}(t)}{\alpha} \int_{X_{6}}\left(\operatorname{tr} \mathcal{R}^{2}\right)^{p} \mathcal{K}
$$

so that

$$
\begin{aligned}
& S I_{p}=-\frac{c_{2(p-1)}(t)}{\alpha} \int_{X_{6}} \mathcal{A}_{4 p} \delta \mathcal{K}=-c_{2(p-1)}(t) \int_{X_{6}} \mathcal{A}_{4 p} \delta_{M_{3}}= \\
& =-c_{2(p-1)}(t) \int_{M_{3}} \mathcal{A}_{4 p}=-S F_{4(p-1)}
\end{aligned}
$$

As mentioned earlier, arguments confirming the presence in the topological closed theory of the term $I_{1}$ which cancels the $p=1$ framing anomaly have been presented in [26]. Here we see that this could be just the tip of an iceberg: To each one of the anomalies of the deformed $S U(N)$ CS theory there should correspond, on the closed string side, a "dressing" of the closed string field by an appropriate topological gravity observable.

\section{Acknowledgements}

I would like to thank M. Bonini, S. Cecotti, S. Giusto and, especially, R. Stora for useful discussions. I am also grateful to the Theory Group of CERN, Geneva, Switzerland, for providing me with hospitality, support and a congenial working environment during the 2008 Institute "Black Holes, a landscape of theoretical physics problems", when part of this work was done.

\section{A Equivariant Cohomology}

In this Appendix we review some standard material about the equivariant cohomology of topological gravity. 
We will be interested in studying cohomologies of $s$ modulo $d$, the exterior differential acting on space-time forms. It is convenient to introduce a fermionic degree $f$ which is the sum of the ghost degree and the form degree. The operators $S_{0}, S, d$ and $i_{\gamma}$ all have $f=+1$. It is therefore coherent to take both $d$ and $i_{\gamma}$ to anti-commute with $S_{0}$ and $S$. With this choice the operator

$$
\tilde{\delta} \equiv s-d
$$

is nilpotent when acting on generalized forms

$$
\tilde{O} \equiv \tilde{O}^{(0)}+\tilde{O}^{(1)}+\tilde{O}^{(2)}+\tilde{O}^{(3)}
$$

$\tilde{\delta}$-cocycles

$$
\tilde{\delta} \tilde{O}=0
$$

are associated to the descent equations

$$
s \tilde{O}^{(3)}=d \tilde{O}^{(2)} \quad s \tilde{O}^{(2)}=d \tilde{O}^{(1)} \quad s \tilde{O}^{(1)}=d \tilde{O}^{(0)} \quad s \tilde{O}^{(0)}=0
$$

and trivial $\tilde{\delta}$-cocycles

$$
\tilde{O}=\tilde{\delta} \tilde{\omega}
$$

correspond to

$$
\begin{array}{ll}
\tilde{O}^{(3)}=s \tilde{\omega}^{(3)}-d \tilde{\omega}^{(2)} & \tilde{O}^{(2)}=s \tilde{\omega}^{(2)}-d \tilde{\omega}^{(1)} \\
\tilde{O}^{(1)}=s \tilde{\omega}^{(1)}-d \tilde{\omega}^{(0)} & \tilde{O}^{(0)}=s \tilde{\omega}^{(0)}
\end{array}
$$

Topological gravity instructs us to consider a particular $s$ cohomology, the equivariant cohomology. This means considering not just any $\tilde{\delta}$-cocycles but those whose dependence on the reparametrization ghost is restricted to be of the following form

$$
\tilde{O}=\mathrm{e}^{i_{\xi}} O
$$

where

$$
O \equiv O^{(0)}+O^{(1)}+O^{(2)}+O^{(3)}
$$

is a generalized form which does not depend on $\xi$. Writing (A.7) in terms of the components of fixed form degree one has

$$
\begin{aligned}
& \tilde{O}^{(3)}=O^{(3)} \\
& \tilde{O}^{(2)}=O^{(2)}+i_{\xi}\left(O^{(3)}\right) \\
& \tilde{O}^{(1)}=O^{(1)}+i_{\xi}\left(O^{(2)}\right)+\frac{1}{2} i_{\xi} i_{\xi}\left(O^{(3)}\right) \\
& \tilde{O}^{(0)}=O^{(0)}+i_{\xi}\left(O^{(1)}\right)+\frac{1}{2} i_{\xi} i_{\xi}\left(O^{(2)}\right)+\frac{1}{3 !} i_{\xi} i_{\xi} i_{\xi}\left(O^{(3)}\right)
\end{aligned}
$$


where $O^{(k)}$, with $k=1,2,3$, are $\xi$-independent.

The following coboundary operator can be defined on the space of $\xi$ independent generalized forms

$$
\delta \equiv S+i_{\gamma}-d
$$

$\delta$ is nilpotent thanks to the relations

$$
S^{2}=\mathcal{L}_{\gamma}=\left\{d, i_{\gamma}\right\}
$$

It is easily shown that equivariant $\tilde{\delta}$-cocycles $\tilde{O}=\mathrm{e}^{i_{\xi}} O$ are in one-to-one correspondence with ( $\xi$-independent) $\delta$-cocycles $O$. The components of fixed form degree of $\delta$-cocycles are in the cohomology of $S$ modulo $d$ and modulo $i_{\gamma}$ :

$$
\begin{aligned}
& S O^{(3)}=d O^{(2)} \\
& S O^{(2)}=d O^{(1)}-i_{\gamma}\left(O^{(3)}\right) \\
& S O^{(1)}=d O^{(0)}-i_{\gamma}\left(O^{(2)}\right) \\
& S O^{(0)}=-i_{\gamma}\left(O^{(1)}\right)
\end{aligned}
$$

Summarizing, the physically relevant equivariant $s$-cohomology modulo $d$ is in a one-to-one correspondence with the $S$-cohomology modulo $d$ and modulo $i_{\gamma}$ on the space of $\xi$-independent operators.

The relation between the $S$-cohomology (modulo $d$ and modulo $i_{\gamma}$ ) and the "naive" $S_{0}$-cohomology (modulo $d$ ) is based on the decomposition

$$
S=S_{0}+G_{\gamma}
$$

which leads to

$$
\delta \equiv \delta_{0}+\delta_{\gamma}
$$

where

$$
\delta_{0} \equiv S_{0}-d \quad \delta_{\gamma}=G_{\gamma}+i_{\gamma}
$$

$\delta_{0}$ and $\delta_{\gamma}$ are (anti)-commuting coboundary operators

$$
\delta_{0}^{2}=\delta_{\gamma}^{2}=\left\{\delta_{\gamma}, \delta_{0}\right\}=0
$$

Since $\delta_{0}$ and $\delta_{\gamma}$ (anti)-commute, we can consider the cohomology of $\delta_{0}$ relative to $\delta_{\gamma}$. This is defined on the $\delta_{\gamma}$-invariant subspace of "matter" operators - i.e. those independent of the gravitational fields. In the context 
of $N=2$ supersymmetric field theories these operators are called "chiral". The cohomology of $\delta_{0}$ relative to $\delta_{\gamma}$ is the kernel of $\delta_{0}$ acting on "chiral" operators, modulo $\delta_{0}$-trivial operators $\delta_{0} \omega$ with $\omega$ "chiral".

A matter operator which is both "chiral" and $\delta_{0}$-closed - i.e. a class in the relative $\delta_{0}$ cohomology - maps, by means of the identity map, to a class of the equivariant $\delta$. In Section 4 we consider such kind of observables.

It should be emphasized that the map between relative $\delta_{0}$-cohomology and equivariant cohomology is not, in general, one-to-one: both its surjectiveness and injectiveness depend on the cohomology of $\delta_{\gamma}$. Only if $\delta_{\gamma}$ had no cohomology, equivariant $\delta$-cohomology and the relative $\delta_{0}$ cohomology would be the same.

\section{B A Cohomological Identity}

We want to show that the differences

$$
\begin{aligned}
\mathcal{B}_{2 k} & \equiv \operatorname{tr} \hat{\mathcal{R}}^{k}-\operatorname{tr} \mathcal{R}^{k}= \\
& =\operatorname{tr} \mathcal{F}^{k}-k \operatorname{tr} \mathcal{F}^{k-1} \mathcal{R}+\cdots
\end{aligned}
$$

are $\delta$-trivial. Let us start with a more general preliminary proposition. Consider an invariant polynomial $P_{k}(\mathcal{F}, \hat{\mathcal{R}}, g)$ of total fermionic degree $2 k$ built with the matrices $\mathcal{F}_{\nu}^{\mu}, \hat{\mathcal{R}}_{\nu}^{\mu}$, and $g^{\mu \nu}$. We will show that if $P_{k}(\mathcal{F}, \hat{\mathcal{R}}, g)$ is $\delta$ closed, then it is cohomologically equivalent to a class which does not contain $\mathcal{F}$.

Let us introduce a grading which counts the numbers of $\mathcal{F}$. We are going to decompose $P_{k}(\mathcal{F}, \hat{\mathcal{R}}, g)$ into components $X_{i}(\mathcal{F}, \hat{\mathcal{R}}, g)$ of fixed $\mathcal{F}$-degree $i$, with $i=0,1, \ldots k$ :

$$
P_{k}(\mathcal{F}, \hat{\mathcal{R}}, g)=X_{k}(\mathcal{F}, \hat{\mathcal{R}}, g)+X_{k-1}(\mathcal{F}, \hat{\mathcal{R}}, g)+\cdots+X_{0}(\hat{\mathcal{R}}, g)
$$

Recall the action of the $\hat{\delta}$-differential (6.27) on the algebra spanned by $\mathcal{C}$, $\mathcal{F}$ and $\hat{\mathcal{R}}$ :

$$
\begin{aligned}
& \hat{\delta} \mathcal{C}=\mathcal{F}+\mathcal{C}^{2} \\
& \hat{\delta} \mathcal{F}=[\mathcal{F}, \mathcal{C}]+[\hat{\mathcal{R}}, \mathcal{C}] \\
& \hat{\delta} \hat{\mathcal{R}}=0
\end{aligned}
$$

Moreover

$$
\hat{\delta} g^{\mu \nu}=-\psi^{\mu \nu}+\frac{1}{2} \psi_{\lambda}^{\mu} g^{\lambda \nu}+\frac{1}{2} \psi_{\lambda}^{\nu} g^{\lambda \mu}=0
$$


Let us decompose $\hat{\delta}$ according to its $\mathcal{F}$-grading:

$$
\hat{\delta}=\hat{\delta}_{1}+\hat{\delta}_{0}+\hat{\delta}_{-1}
$$

$\hat{\delta}_{m}$ for $m=-1,0,1$ have $\mathcal{F}$-degree equal to $m$ :

$$
\begin{array}{ll}
\hat{\delta}_{1} \mathcal{C}=\mathcal{F} & \hat{\delta}_{1} \mathcal{F}=0 \\
\hat{\delta}_{0} \mathcal{C}=\mathcal{C}^{2} & \hat{\delta}_{0} \mathcal{F}=[\mathcal{F}, \mathcal{C}] \\
\hat{\delta}_{-1} \mathcal{C}=0 & \hat{\delta}_{-1} \mathcal{F}=[\hat{\mathcal{R}}, \mathcal{C}] \\
\hat{\delta}_{m} \hat{\mathcal{R}}=\hat{\delta}_{m} g=0 \quad \text { for } m=-1,0,1
\end{array}
$$

One verifies that

$$
\hat{\delta}_{1}^{2}=\hat{\delta}_{0}^{2}=\hat{\delta}_{-1}^{2}=\left\{\hat{\delta}_{0}, \hat{\delta}_{1}\right\}=\left\{\hat{\delta}_{0}, \hat{\delta}_{-1}\right\}=0
$$

and

$$
\left\{\hat{\delta}_{1}, \hat{\delta}_{-1}\right\} X=[\hat{\mathcal{R}}, X]
$$

on any matrix-valued generalized form $X$.

Decomposing the equation

$$
\hat{\delta} P_{k}(\mathcal{F}, \hat{\mathcal{R}}, g)=0
$$

in components of fixed $\mathcal{F}$-degree one derives the descent equations

$$
\begin{aligned}
& \hat{\delta}_{1} X_{k}=0 \\
& \hat{\delta}_{1} X_{k-1}+\hat{\delta}_{0} X_{k}=0 \\
& \hat{\delta}_{1} X_{k-2}+\hat{\delta}_{0} X_{k-1}+\hat{\delta}_{-1} X_{k}=0 \\
& \ldots \\
& \hat{\delta}_{1} X_{0}+\hat{\delta}_{0} X_{1}+\hat{\delta}_{-1} X_{2}=0 \\
& \hat{\delta}_{0} X_{0}+\hat{\delta}_{-1} X_{1}=0 \\
& \hat{\delta}_{-1} X_{0}=0
\end{aligned}
$$

The basic observation is that the nilpotent $\hat{\delta}_{1}$ has, obviously, vanishing cohomology on sectors with non-zero $\mathcal{F}$-degree. Therefore, from the first of the equations (B.10) one deduces

$$
X_{k}=\hat{\delta}_{1} Y_{k-1}
$$


which inserted in the second equation gives

$$
\hat{\delta}_{1}\left(X_{k-1}-\hat{\delta}_{0} Y_{k-1}\right)=0
$$

This in turns implies

$$
\begin{aligned}
& X_{k-1}=\hat{\delta}_{1} Y_{k-2}+\hat{\delta}_{0} Y_{k-1} \\
& X_{k-2}=\hat{\delta}_{1} Y_{k-3}+\hat{\delta}_{0} Y_{k-2}+\hat{\delta}_{-1} Y_{k-1}
\end{aligned}
$$

and so on, until one reaches the equation:

$$
X_{0}=\hat{\delta}_{0} Y_{0}+\hat{\delta}_{-1} Y_{1}+Z(\hat{\mathcal{R}}, g)
$$

where $Z(\hat{\mathcal{R}}, g)$ does not depend on $\mathcal{F}$. Putting (B.13) together with the previous equations one finally obtains

$$
P_{k}(\mathcal{F}, \hat{\mathcal{R}}, g)=\hat{\delta}\left(Y_{0}+Y_{1}+\cdots+Y_{k-1}\right)+Z(\hat{\mathcal{R}}, g)
$$

which is our preliminary proposition. Let us apply it to $\mathcal{B}_{2 k}$. In this case the component of zero $\mathcal{F}$-degree vanishes

$$
X_{0}=0=\hat{\delta}_{0} Y_{0}+\hat{\delta}_{-1} Y_{1}+Z(\hat{\mathcal{R}}, g)
$$

But both $\hat{\delta}_{0}$ and $\hat{\delta}_{-1}$ increase the number of $\mathcal{C}$ by one. Therefore

$$
\hat{\delta}_{0} Y_{0}+\hat{\delta}_{-1} Y_{1}=0
$$

and

$$
Z(\hat{\mathcal{R}}, g)=0
$$

which is what we wanted to show.

\section{References}

[1] A. S. Schwarz, Lett. Math. Phys. 2 (1978) 247.

J. F. Schonfeld, Nucl. Phys. B 185 (1981) 157.

R. Jackiw and S. Templeton, Phys. Rev. D 23 (1981) 2291.

S. Deser, R. Jackiw and S. Templeton, Phys. Rev. Lett. 48 (1982) 975.

S. Deser, R. Jackiw and S. Templeton, Annals Phys. 140 (1982) 372; Annals Phys. 185 (1988) 406, Erratum. 
[2] E. Witten, Commun. Math. Phys. 121 (1989) 351.

[3] D. Bar-Natan and E. Witten, Commun. Math. Phys. 141, 423 (1991).

[4] E. Witten, Prog. Math. 133 (1995) 637.

[5] H. Kluberg-Stern and J. B. Zuber, Phys. Rev. D 12 (1975) 467.

H. Kluberg-Stern and J. B. Zuber, Phys. Rev. D 12 (1975) 482.

H. Kluberg-Stern and J. B. Zuber, Phys. Rev. D 12 (1975) 3159.

O. Piguet and K. Sibold, Nucl. Phys. B 253 (1985) 517.

[6] O. Piguet and K. Sibold, Nucl. Phys. B 248 (1984) 301.

[7] C. M. Becchi and C. Imbimbo, Nucl. Phys. B 462 (1996) 571.

[8] E. Witten, Phys. Lett. B 206 (1988) 601.

[9] L. Baulieu and I. M. Singer, Nucl. Phys. Proc. Suppl. 5B (1988) 12.

[10] S. Ouvry, R. Stora and P. van Baal, Phys. Lett. B 220 (1989) 159.

[11] L. Baulieu and I. M. Singer, Commun. Math. Phys. 125 (1989) 227.

[12] H. Kanno, Z. Phys. C 43 (1989) 477.

[13] L. Baulieu, G. Bossard and A. Tanzini, JHEP 0508 (2005) 037.

[14] A. Fuster, M. Henneaux and A. Maas, Int. J. Geom. Meth. Mod. Phys. 2 (2005) 939.

[15] F. Delduc, F. Gieres and S. P. Sorella, Phys. Lett. B 225 (1989) 367.

[16] D. Birmingham, M. Rakowski and G. Thompson, Nucl. Phys. B 329 (1990) 83.

[17] F. Delduc, C. Lucchesi, O. Piguet and S. P. Sorella, Nucl. Phys. B 346 (1990) 313.

[18] C. Lucchesi, O. Piguet and S. P. Sorella, Nucl. Phys. B 395 (1993) 325.

[19] A. Brandhuber, M. Langer, M. Schweda, O. Piguet and S. P. Sorella, Phys. Lett. B 300 (1993) 92 arXiv:hep-th/9212112. 
[20] M. Dubois-Violette, M. Talon and C. M. Viallet, Commun. Math. Phys. 102 (1985) 105.

[21] S. Axelrod and I. M. Singer, J. Diff. Geom. 39 (1994) 173.

[22] M. Alexandrov, M. Kontsevich, A. Schwartz and O. Zaboronsky, Int. J. Mod. Phys. A 12 (1997) 1405.

[23] R. C. Myers and V. Periwal, Nucl. Phys. B 333 (1990) 536.

[24] R. C. Myers, Nucl. Phys. B 343 (1990) 705.

[25] M. Bershadsky and V. Sadov, Int. J. Mod. Phys. A 11 (1996) 4689.

[26] R. Gopakumar and C. Vafa, Adv. Theor. Math. Phys. 3 (1999) 1415.

[27] M. Kontsevich, Commun. Math. Phys. 147 (1992) 1.

[28] D. Gaiotto and L. Rastelli, JHEP 0507, 053 (2005). 